\title{
The Thermodynamic Model for Nuclear Multifragmentation
}

\author{
C. B. Das ${ }^{a, b}$ S. Das Gupta ${ }^{a, 1}$ W. G. Lynch $^{c}$ A. Z. Mekjian ${ }^{d}$ \\ M. B. Tsang ${ }^{\mathrm{c}, 2}$
}

${ }^{a}$ Physics Department, McGill University, Montréal, Canada H3A 2T8

${ }^{\mathrm{b}}$ Physics Division, Variable Energy Cyclotron Centre, Kolkata 700 064, India

${ }^{\mathrm{c}}$ National Superconducting Cyclotron Laboratory and Physics and Astronomy

Department, Michigan State University, East Lansing, MI 48824, USA

d Physics Deptartment, Rutgers University, Piscataway, NJ 08854, USA 


\section{Contents}

1 Introduction 4

2 The Basic Formulae $\quad 6$

3 General features of yields of composites 11

4 Phase transition in the model $\quad 11$

4.1 Signatures from thermodynamic variables . . . . . . . . . . . . 11

4.2 Power-law and scaling behaviour of composite yields . . . . . . . . 13

4.3 Comparision with mean-field theory . . . . . . . . . . . 18

5 Comparison of canonical and grand canonical 20

6 Specific heat at constant pressure $\quad 26$

7 Corrections for secondary decay $\quad 32$

7.1 Levels and level densities . . . . . . . . . . . . . . . . . 32

7.2 Sequential decay algorithm . . . . . . . . . . . . . . 34

8 Comparisons to data $\quad \mathbf{3 5}$

8.1 Charge and Mass Distributions . . . . . . . . . . . . 36

8.2 Isotopic distributions . . . . . . . . . . . . . . . . . . . . . 39

8.3 Isoscaling . . . . . . . . . . . . . . . . . . . . 41

8.4 Isotopic temperatures . . . . . . . . . . . . . . . 44

$\begin{array}{lll}9 & \text { Summary } & 47\end{array}$

$\begin{array}{ll}10 \text { Acknowledgement } & 47\end{array}$

A Equilibrium, reactions and reaction rate time scales $\quad 47$

B Antisymmetry and all that $\quad 50$

$\begin{array}{lll}\text { C Applications to other areas } & 53\end{array}$

$\begin{array}{ll}\text { References } & 56\end{array}$

List of Figures 


\begin{abstract}
A great many observables seen in intermediate energy heavy ion collisions can be explained on the basis of statistical equilibrium. Calculations based on statistical equilibrium can be implemented in microcanonical ensemble (energy and number of particles in the system are kept fixed), canonical ensemble (temperature and number of particles are kept fixed) or grand canonical ensemble (fixed temperature and a variable number of particles but with an assigned average). This paper deals with calculations with canonical ensembles. A recursive relation developed recently allows calculations with arbitrary precision for many nuclear problems. Calculations are done to study the nature of phase transition in intermediate energy heavy ion collision, to study the caloric curves for nuclei and to explore the possibility of negative specific heat because of the finiteness of nuclear systems. The model can also be used for detailed calculations of other observables not connected with phase transitions, such as populations of selected isotopes in a heavy ion collision.

The model also serves a pedagogical purpose. For the problems at hand, both the canonical and grand canonical solutions are obtainable with arbitrary accuracy hence we can compare the values of observables obtained from the canonical calculations with those from the grand canonical. Sometimes, very interesting discrepancies are found.

To illustrate the predictive power of the model, calculated observables are compared with data from the central collisions of Sn isotopes.
\end{abstract}

Key words: heavy ion intermediate energy composites multiplicity PACS: 25.70.-z 25.75.Ld 25.10.Lx

1 E-mail: dasgupta@physics.mcgill.ca

2 E-mail: tsang@nscl.msu.edu 


\section{Introduction}

Consider a central collision of two heavy ions. Nucleons from one nucleus will collide with nucleons from another nucleus. After a few collisions a given nucleon may lose the identity of its source. The system then more resembles a hot fluid of nucleons in an overall volume. Depending upon the original beam energy, this system may undergo an initial compression and then begins to decompress. During this time the nucleons will interact with each other, at least between the nearest neighbours. As the density of the system decreases, higher density regions will develop into composites. As this collection of nucleons begin to move outwards, rearrangements, mass transfers, nucleon coalescence and most physics will continue to happen until the density decreases so much that the mean free paths for such processes become larger than the dimension of the system. Subsequently the objects follow the long range coulomb trajectories. Our objective is to have a soluble model which describes the physics of the situation at this freezeout density when one averages many nucleus-nucleus collisions.

Although we chose central collisions to describe this scenario a similar situation will arise even for semi-central or semi-peripheral collisions. In such cases one may have projectile like fragment (and target like fragment and participants, region of violent collisions). For example, a projectile fragment may be excited which resembles a system of hot particles whose centre of mass velocity is close to that of the projectile [1].

The central assumption of the present article (and many others) is that equilibrium statistical mechanics can be used to describe the hot fluid of nucleons. Even the most well prepared experimental measurement of an energetic nucleus - nucleus collision represents an average of a very large number of initial states. In addition to this large number of different initial states, a large number of nucleon - nucleon collisions occur within each nucleus nucleus collision. Together, this means that for many experimental observables almost all the relevant phase-space can be opened up and described by the microcanonical ensemble in which the probability of reaching a channel $y$ is $\Omega(y) / \sum_{y} \Omega(y)$. Here $\Omega(y)$ is the phase-space volume in the channel $y$. In the canonical ensemble the corresponding expression [2] is writen as: $\exp (-f(y) / T) / \sum \exp (-f(y) / T)$. Here $f(y)$ is the free energy in the channel $y$. Since $f(y)=-T \ln Q(y)$ where $Q(y)$ is the canonical partition function in the channel $y$, an equivalent expression is $Q(y) / \sum Q(y)$. A more detailed discussion of statistical equilibrium using reaction rates is given in Appendix A.

The obvious experimental observables in heavy ion collisions are the number of nucleons and composites and their velocity distributions that result af- 
ter the collision. The calculation of these in equilibrium statistical mechanics for Bevalac physics is more than twenty five years old [3,4,5]. At that time, the grand canonical ensemble was used to describe data from the Bevalac which normally used beam energies higher than $250 \mathrm{MeV} /$ nucleon. However, at these energies most of the subtle and interesting features of equilibrium statistical mechanics as it pertains to heavy ion collision have disappeared. As the cross-sections of composites fall rapidly with $A$, the mass number, the most interesting results were productions of new particles such as pions and kaons, which can be included in the statistical model. Some discussion of this production is also given in Appendices A and C. Even so, Bevalac experiments brought out beautiful features of dynamics and established narrow limits on compressibility of nuclear matter and the momentum dependence of the real part of the optical potential.

The applications of equilibrium statistical mechanics for intermediate energy heavy ion collisions started in the eighties. At these energies, the efforts switched to microcanonical ensembles although the concept of temperature was sometimes used $[6,7,8]$. One model called the Copenhagen SMM (statistical multifragmentation model) is frequently used [8]. Another popular model is the Berlin model [7]. The use of the canonical ensemble, the main topic of this paper, is more recent[9]. It is as easy to implement as the grand canonical (and more accurate since fluctuations in the number of particles are eliminated: these sometimes cause large errors in computations of observables). It is orders of magnitudes simpler than the microcanonical ensemble although in the latter more fine tuning can be done. These fine tunings do not appear important for most observables.

What are the important issues we want to learn about in intermediate energy heavy ion collisions? For many, it is to extract from data signals of a liquid-gas phase transition in nuclear matter. Nuclear matter is a hypothetical large chunk of matter with $N=Z$ where the coulomb interaction has been switched off. The $p-V$ diagram for nuclear matter with reasonable forces looks like a Van der Waals equation of state [10]. One would then expect to see a liquid-gas phase transition if the experimental conditions are optimal. Such optimal conditions are discussed by Curtin, Toki and Scott [11] and Bertsch and Siemens [12]. For Bevalac energies the evolution of the temperature would go above the phase transition temperature but accelerators at the National Superconducting Cyclotron Laboratory (NSCL), the Texas A\&M cyclotron, the Grand Accelerateur National D'ions Lourds (GANIL) and at Gesellschaft fur Schwerionenforschung $\mathrm{mbH}$ (GSI) can reach the liquid-gas phase transition region and offer the best possibility for experimental study. Further details of theoretical considerations which prompt an experimental investigation of the liquid-gas phase transition can be found in [13].

Unfortunately, this investigation of liquid-gas phase transition in intermediate 
energy heavy ion collisions is fraught with many difficulties. Phase transitions occur in very large systems. In nuclear collisions, we are limited to three to four hundred nucleons (sometimes much less). For finite systems, signals of phase transition get diluted and distinctions between first order and second order transitions get blurred. The coulomb interaction, which prevents large nuclei from forming also interferes with the signals. It is thus necessary to use theories to clarify the situation. If one has a theory which fits many data, not necessarily related to phase transitions, but in addition predicts a phase transition one has some hope for the model to be valid. In this paper we will discuss phase transitions but in addition, data which will be compared to the thermodynamic model predictions.

\section{The Basic Formulae}

This section sets up the basic formulae of the model $[9,14]$.

If there are $A$ identical particles of only one kind in an enclosure at temperature $T$, the partition function of the system can be written as

$$
Q_{A}=\frac{1}{A !}(\omega)^{A}
$$

Here $\omega$ is the partition function of one particle. For a spinless particle without any internal structure $\omega=\frac{V}{h^{3}}(2 \pi m T)^{3 / 2} ; m$ is the mass of the particle; $V$ is the available volume within which each particle moves; $A$ ! corrects for Gibb's paradox. If there are many species, the generalisation is

$$
Q_{A}=\sum \prod_{i} \frac{\left(\omega_{i}\right)^{n_{i}}}{n_{i} !}
$$

Here $\omega_{i}$ is the partition function of a composite which has $i$ nucleons. For a dimer $i=2$, for a trimer $i=3$ etc. Eq. (2) is no longer trivial to calculate. The trouble is with the sum in the right hand side of eq. (2). The sum is restrictive. We need to consider only those partitions of the number $A$ which satisfy $A=\sum i n_{i}$. The number of partitions which satisfies the sum is enormous. We can call a given allowed partition to be a channel. The probablity of the occurrence of a given channel $P(\vec{n}) \equiv P\left(n_{1}, n_{2}, n_{3} \ldots\right)$ is

$$
P(\vec{n})=\frac{1}{Q_{A}} \prod \frac{\left(\omega_{i}\right)^{n_{i}}}{n_{i} !}
$$


The average number of composites of $i$ nucleons is easily seen from the above equation to be

$$
\left\langle n_{i}\right\rangle=\omega_{i} \frac{Q_{A-i}}{Q_{A}}
$$

Since $\sum i n_{i}=A$, one readily arrives at a recursion relation [15]

$$
Q_{A}=\frac{1}{A} \sum_{k=1}^{A} k \omega_{k} Q_{A-k}
$$

For one kind of particle, $Q_{A}$ above is easily evaluated on a computer for $A$ as large as 3000 in matter of seconds. It is this recursion relation that makes the computation so easy in the model. Of course, once one has the partition function all relevant thermodynamic quantities can be computed.

We now need an expression for $\omega_{k}$ which can mimic the nuclear physics situation. We take

$$
\omega_{k}=\frac{V}{h^{3}}(2 \pi m T)^{3 / 2} \times q_{k}
$$

where the first part arises from the centre of mass motion of the composite which has $k$ nucleons and $q_{k}$ is the internal partition function. For $k=1$, $q_{k}=1$ and for $k \geq 2$ it is taken from the Fermi-gas model. For each composite consisting of $k$ nucleons, we approximate the intrinsic free energy at freeze-out by $E-T S=-W_{0} k+\sigma(T) k^{2 / 3}+k T^{2} / \epsilon_{0}-T \times 2 k T / \epsilon_{0}$ where $\epsilon_{0}$ is a constant. This gives

$$
q_{k}=\exp \left[\left(W_{0} k-\sigma(T) k^{2 / 3}+T^{2} k / \epsilon_{0}\right) / T\right]
$$

Here, as in [8], $W_{0}=16 \mathrm{MeV}$ is the volume energy term, $\sigma(T)$ is a temperature dependent surface tension term. The value of $\epsilon_{0}$ is taken to be $16 \mathrm{MeV}$. The explicit expression for $\sigma(T)$ used here, as in [8], is

$\sigma(T)=\sigma_{0}\left[\left(T_{c}^{2}-T^{2}\right) /\left(T_{c}^{2}+T^{2}\right)\right]^{5 / 4}$

with $\sigma_{0}=18 \mathrm{MeV}$ and $T_{c}=18 \mathrm{MeV}$. In the nuclear case one might be tempted to interpret $V$ of eq.(6) as simply the freeze-out volume but it is clearly less than that; $V$ is the volume available to the particles for the centre of mass motion. Assume that the only interaction between clusters is that they can not overlap one another. Then in the Van der Waals spirit we take $V=$ $V_{\text {freeze }}-V_{e x}$ where $V_{e x}$ is taken here to be constant and equal to $V_{0}=A / \rho_{0}$. The assumption that the interaction between different composites is only reflected 
through an excluded volume and that this excluded volume is independent of multiplicity is an idealisation which will fail for a non-dilute system. We therefore restrict the model, somewhat arbitrarily to volumes $V_{\text {freeze }} \geq 2 V_{0}$. There are experimental signatures that $V_{\text {freeze }}$ is indeed greater than $2 V_{0}$ [13] so this is not a debilitating feature of the model. In all our considerations we restrict $\rho / \rho_{0}$ to less than 0.5 .

Among quantities of interest is the inclusive cross-section given by eq.(4). Actually this is a simplification. The occupation given by eq.(4) is the occupation of the composite with $i$ nucleons at temperature $T$. Both the ground state and the excited states contribute to $\left\langle n_{i}\right\rangle$. Some of the excited states will be particle unstable and will decay into lower mass composites before they reach the detector. On the other hand, some higher mass composites, will, by the same argument, decay into the composite $i$. In later sections, where we compare populations with data, this aspect will be taken care of. The expression for $E$ at a given temperature $T$ is simple (this is needed for a caloric curve which is measured in experiments). The energy carried by one composite is given by

$E_{k}=T^{2} \partial \ln \omega_{k} / \partial T=\frac{3}{2} T+k\left(-W_{0}+T^{2} / \epsilon_{0}\right)+\sigma(T) k^{2 / 3}-T[\partial \sigma(T) / \partial T] k^{2 / 3}$.

Of these the first term comes from the centre of mass motion and the rest from $q_{k}$. The term $T[\partial \sigma(T) / \partial T] k^{2 / 3}$ comes from the temperature dependence of the surface tension. It has a small effect. The energy of the whole system is given by $E=T^{2} \frac{1}{Q_{A}} \frac{\partial Q_{A}}{\partial T}$. Using eqs.(2) and (4) we arrive at a very transparent formula $: E=\sum\left\langle n_{k}\right\rangle E_{k}$. The pressure is given by $p=T \partial \ln Q_{A} / \partial V$. If for purposes of illustration, we neglect the long range Coulomb interactions and use eqs.(2) and (4) we get $p=T \frac{1}{V} \sum\left\langle n_{i}\right\rangle$. This is just the law of partial pressures.

For analysing phase transitions in the model, it is very useful to calculate the average value of the largest cluster in the ensemble. Eq. (2) shows that the size of the largest cluster varies. In that ensemble there is a term $\frac{\omega_{1}^{A}}{A !}$. For this the largest cluster is the monomer. For example in eq.(2) we also have a term $\frac{\omega_{1}^{n}}{n !} \frac{\omega_{2}^{(A / 2-n / 2)}}{(A / 2-n / 2) !}$. Here the largest cluster is the dimer. Consider building $Q_{A}$ with $\omega_{1}, \omega_{2} \ldots \ldots \omega_{k}, 0,0,0,0 \ldots$ In this ensemble the largest cluster will span from monomer upto a composite with $k$ nucleons. Let us label this partition function $Q_{A}\left(\omega_{1}, \omega_{2}, \ldots \omega_{k}, 0,0,0 ..\right)$. Let us also build a $Q_{A}$ where the largest non-zero $\omega$ is $\omega_{k-1}$. The partition function is $Q_{A}\left(\omega_{1}, \omega_{2}, . . \omega_{k-1}, 0,0,0,0\right)$. In this ensemble all the previous channels are included except where the largest cluster had $k$ nucleons. If we define

$$
\Delta Q_{A}(k)=Q_{A}\left(\omega_{1}, \omega_{2} \ldots \omega_{k}, 0,0, . .\right)-Q_{A}\left(\omega_{1}, \omega_{2} \ldots \omega_{k-1}, 0,0 \ldots\right),
$$

then the probability of the largest cluster having $k$ nucleons is 


$$
\operatorname{Pr}(k)=\frac{\Delta Q_{A}(k)}{Q_{A}\left(\omega_{1}, \omega_{2} \ldots \ldots \omega_{A}\right)}
$$

If we now label the average value of the largest cluster as $\left\langle k_{\max }\right\rangle$, then $\left\langle k_{\max }\right\rangle=$ $\sum k \times \operatorname{Pr}(k)$. A more useful quantity is $\frac{\left\langle k_{\max }\right\rangle}{A}$. The limits of this are $\approx 0$ and 1 .

Another interesting quantity which has been the subject of an enormous amount of interpretation [17] is the multiplicity distribution of a species or a group of species. In most models this requires a very elaborate Monte-Carlo calculation. In the canonical ensemble there is an elegant equation.

$$
P_{n}(k)=\frac{1}{Q_{A}} \frac{\omega_{k}^{n}}{n !} Q_{A-n k}\left(\omega_{1}, \omega_{2} \ldots \omega_{k-1}, \omega_{k}=0, \omega_{k+1} . . \omega_{A}\right)
$$

Here $P_{n}(k)$ is the probability of obtaining the composite $k$ n times.

The strength of the canonical model as described here lies in the fact that all calculations above avoid Monte-Carlo sampling. In many other models, a Monte-Carlo sampling over the channels is required. Since the number of channels is enormous, this requires great ingenuity but also much more computer time.

The model of one kind of particles in which composites have a volume energy, a surface energy and excited states is already very useful for investigations of phase transition, caloric curves etc. and we will pursue this in latter sections a great deal. Let us nonetheless introduce here the model with two kinds of particles (so that one can compare with actual nuclear cases) $[13,14,16]$. Now a composite is labelled by two indices $\omega \rightarrow \omega_{i, j}$. The partition function for a system with $Z$ protons and $N$ neutrons is given by

$$
Q_{Z, N}=\sum \prod_{i, j} \frac{\omega_{i, j}^{n_{i, j}}}{n_{i, j} !}
$$

There are two constraints: $Z=\sum i \times n_{i, j}$ and $N=\sum j \times n_{i, j}$. These lead to two recursion relations any one of which can be used. For example

$$
Q_{Z, N}=\frac{1}{Z} \sum_{i, j} i \omega_{i, j} Q_{Z-i, N-j}
$$

where

$$
\omega_{i, j}=\frac{V}{h^{3}}(2 \pi m T)^{3 / 2}(i+j)^{3 / 2} \times q_{i, j}
$$


Here $q_{i, j}$ is the internal partition function. These could be taken from experimental binding energies, excited states and some model for the continuum or from the liquid drop model in combination with other models. The versatility of the method lies in being able to accommodate any choice for $q_{i, j}$. A choice of $q_{i, j}$ from a combination of the liquid drop model for binding energies and the Fermi-gas model for excited states that has been used is

$$
q_{i, j}=\exp \frac{1}{T}\left[W_{0} a-\sigma a^{2 / 3}-\kappa \frac{i^{2}}{a^{1 / 3}}-s \frac{(i-j)^{2}}{a}+T^{2} a / \epsilon_{0}\right]
$$

where $a=i+j, W_{0}=15.8 \mathrm{MeV}, \sigma=18.0 \mathrm{MeV}, \kappa=0.72 \mathrm{MeV}, s=23.5 \mathrm{MeV}$ and $\epsilon_{0}=16 \mathrm{MeV}$. One can recognise in the parametrisation above, the volume term, the surface tension term, the coulomb energy term, the symmetry energy term and contributions from excited states.

The coulomb interaction is long range. Some effects of the coulomb interaction between different composites can be included in an approximation called the Wigner-Seitz approximation. We assume, as usual, that the break up into different composites occurs at a radius $R_{c}$, which is greater than the normal radius $R_{0}$. Considering this as a process in which a uniform dilute charge distribution within radius $R_{c}$ collapses successively into denser blobs of proper radius $R_{i, j}$, we write the coulomb energy as [8]

$$
E_{C}=\frac{3}{5} \frac{Z^{2} e^{2}}{R_{c}}+\sum_{i, j} \frac{3}{5} \frac{i^{2} e^{2}}{R_{i, j}}\left(1-R_{0} / R_{c}\right)
$$

It is seen that the expression is correct in two extreme limits: very large freezeout volume $\left(R_{c} \rightarrow \infty\right)$ or if the freeze out volume is the normal nuclear volume so that one has just one nucleus with the proper radius.

For the thermodynamic model that we have been pursuing, the constant term $\frac{3}{5} \frac{Z^{2} e^{2}}{R_{c}}$ is of no significance since the freeze-out volume is assumed to be constant. In a mean-field sense then one would just replace the coulomb term in eq. (13) by $\kappa \frac{i^{2}}{a^{1 / 3}}\left(1.0-\left(\rho / \rho_{0}\right)^{1 / 3}\right)$.

Before we leave this section, we mention that the mass parametrisation implied by eq.(13) can be vastly improved with only slight complications. We will later present results with the improved formula [16]. A pedagogical issue: although we have derived results here based on eq.(1) which takes care of (anti)symmetrisation only approximately it is shown in [18] that the specific structure of eqs.(5) and (11) occur more generally when (anti)symmetrisation is included properly. Part of this argument is presented in appendix B which also demonstrates that results based on this section are quite accurate. 


\section{General features of yields of composites}

We pursue here the model of one kind of particles. For 200 particles at a constant freeze-out volume $=3.7 V_{0}$ we have plotted in fig. $1\left\langle n_{k}\right\rangle$ (in the figure we call this $Y(a)=$ yield of composite of mass $a$ ) at three temperatures. At the lowest temperature shown the curve has a $U$ shape. The yields $Y(a)$ first begin to fall, then reach a minimum and then the yields for heavier masses increase finally cutting off at 200. In the literature the heavy fragments are called the liquid phase. The light fragments are gas particles. As the temperature increases the maximum at the higher $a$ decreases in height finally disappearing at $\sim 6.35 \mathrm{MeV}$. At higher temperature $Y(a)$ falls monotonically. The surface tension plays a crucial role in this evolution. At any temperature the lowest value of the free energy $E-T S$ will be obtained. It costs in the energy term $E$ to break up a system. A nucleus of $A$ nucleons has less surface than the total surface of two nuclei each of $A / 2$ nucleons (the volume energy term has no preference between the two alternatives). Therefore at low temperature one will see a large chunk. The - TS term favours break up into small objects. The competition between these two effects leads to the general features of $Y(a)$ as

a function of temperature. As we will see in the next section, the temperature at which the maximum of the yield at the high side of $a$ disappears is the phase transition temperature.

Similar features are seen also in other models of multifragmentation as applied to nuclear physics. The earliest such model was the percolation model $[19,20]$. The model has a parameter $p$ which gives the probability of two nearest neighbour sites joining together as in a composite. Beyond a certain value of $p$, a percolating cluster is formed which goes from edge to edge of the system. This corresponds to the large cluster which forms at the lower temperature in fig.1. The lattice gas model [21] has similarity with the percolation model but has a Hamiltonian, includes percolation model as a subset [22] and also includes the formation of a percolating cluster

\section{Phase transition in the model}

\subsection{Signatures from thermodynamic variables}

We now begin the discussion of a phase transition in the model. The free energy of a system of particles is given by $F=-T \ln Q_{A}$ and $\ln Q_{A}$ is directly calculable with eq.(5). For a system of 200 and 2000 particles, the free energy per particle is shown in the top panel of fig.2, as a function of temperature for fixed freezeout density $0.27 \rho_{0}$. An approximate break in the first derivative of $F / A$ is 
seen to develop at $\approx 6.35 \mathrm{MeV}$ for 200 particles and at $\approx 7.15 \mathrm{MeV}$ for 2000 particles. We believe the break would be rigorous if we could go to an infinite system. A break in the first derivative implies a first order phase transition and a discontinuous change in the value of entropy per particle. This would imply that the specific heat at constant volume per particle $c_{v}=\left(\frac{\partial(E / A)}{\partial T}\right)_{V}$ would go through a peak (for an infinite system this peak would go to $\infty$ ). We show this in the midddle panel of fig. 2 for systems of 200 and 2000 particles, where we find the width of the peak decreases and the height of the peak increases as the particle number increases. As expected, the temperature where the specific heat maximises also coincides with the temperature at which the maximum in the high side of $a$ (fig.1) just disappears.

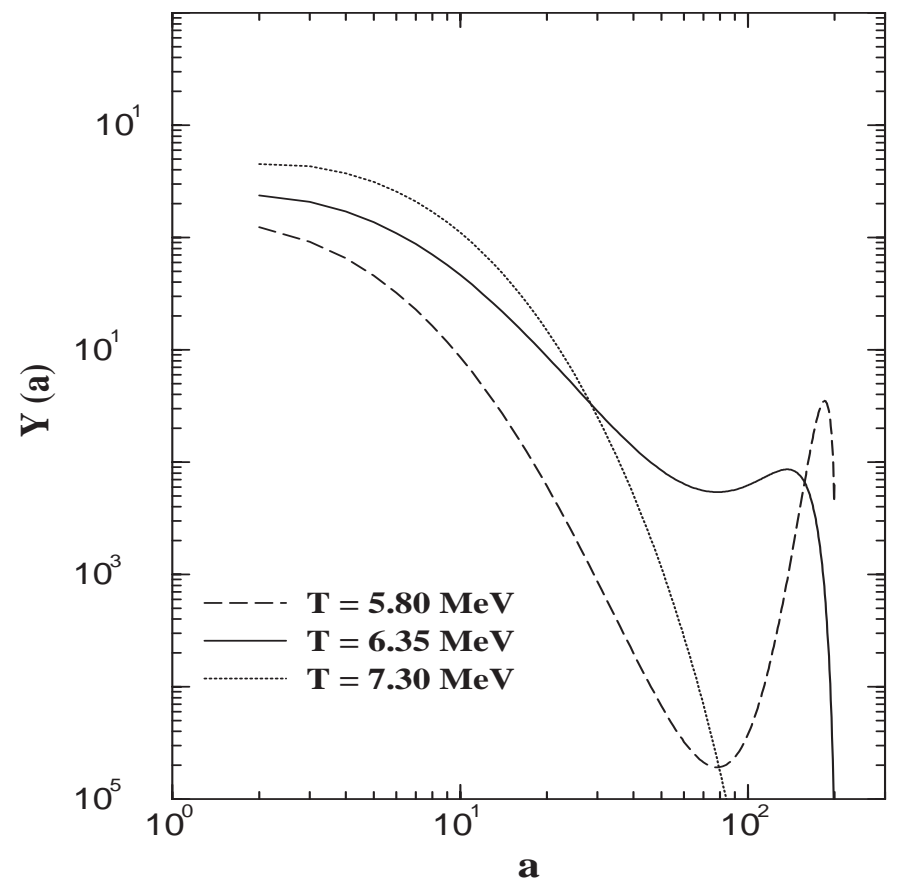

Fig.1 Yield $Y(a)$ against $a$ at three different temperatures. The dissociating system has 200 particles and the freeze-out density is $0.27 \rho_{0}$.

Another very interesting quantity is the quantity $\left\langle k_{\max }\right\rangle / A$ (i.e. the size of the largest cluster) as the temperature varies. This can be calculated using eq.(8).We define $T_{b}$ as the temperature where the break in the derivative of the free energy occurs (this is the first order phase transition temperature). Calculating the size of the largest cluster at different temperatures, we find that $\left\langle k_{\max }\right\rangle / A$ approaches 1 as $T<T_{b}$ and approaches a small number as $T>T_{b}$. The change is smooth for low mass nuclei (bottom panel of fig.2) but becomes more sudden for larger systems. For large systems there is a large blob (i.e., liquid) below $T_{b}$ which disappears as soon as $T$ crosses $T_{b}$. This we think is a very engaging example of boiling emerging from a theoretical 
calculation.

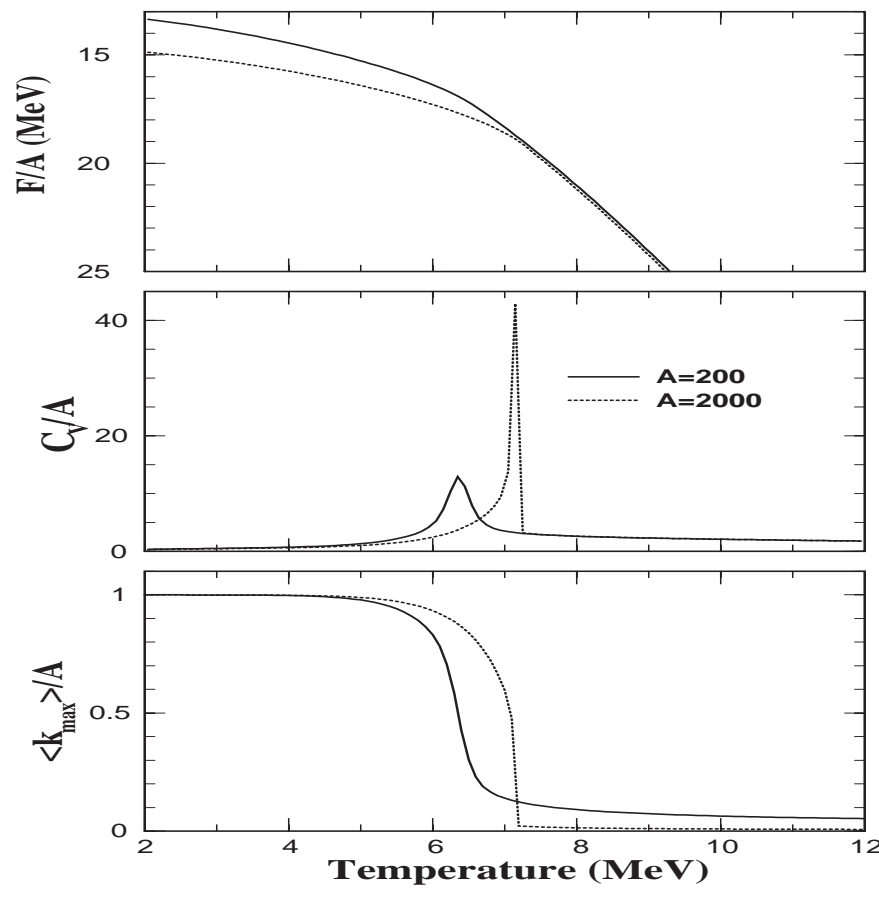

Fig. 2. The free-energy per particle, the specific heat at constant volume, $C_{V} / A$ and the size of the largest cluster as a function of temperature, systems of 200 and 2000 particles.

To summarise, the thermodynamic model predicts unequivocally a first order phase transition at intermediate energy. In the realm of density $\rho / \rho_{0} \leq 0.5$ for which we believe the model to be reasonable there is no critical point. Bugaev et al. [23] have taken the model beyond this range of density and find that the critical density is $\rho / \rho_{0}=1$ and the temperature is $18 \mathrm{MeV}$ when the surface tension $\sigma(T)$ goes to zero. We end this section by noting that microcanonical calculations using statistical equilibrium were also suggestive of a first order phase transition occurring at intermediate energy $[24,6]$.

\subsection{Power-law and scaling behaviour of composite yields}

A rather large part of literature in heavy ion reaction postulates that in multifragmentation at intermediate energy one is near the critical point of nuclear matter. One then proceeds to determine from the data the critical temperature and various critical exponents. The working formula, obtained from models of critical phenomena (to see how the formula arises, refer to $[25,26]$ ) is 


$$
\left\langle n_{a}\right\rangle=a^{-\tau} f\left(a^{\sigma}\left(T-T_{c}\right)\right)
$$

Here $\tau$ is called the Fisher exponent [27], $a$ is the mass number of the composite, $\sigma$ is a critical exponent and $T_{c}$ the critical temperature ; $f$ is as yet an unspecified function but instead of being a general function of $a$ and $T$ it is a function only of the combination $a^{\sigma}\left(T-T_{c}\right)$. This is called scaling. At $T=T_{c}$ the yield $\left\langle n_{a}\right\rangle=a^{-\tau} f(0)$ is a pure power law but away from $T_{c}$ it will deviate from a power law.

In intermediate energy collisions, even if we proceed under the assumption that one is observing critical phenomena we can not expect near perfect fit to eq.(15) whose validity depends upon the dissociating system being very large. Also the range of $a$ is to be chosen judiciously. It can not be very small (since eq.(15) applies to "large" $a$ 's [25]). But $a$ also should be truncated on the high side (significantly smaller than the size of the dissociating system).

With these provisos we can at best expect a moderately good fit. Extracting $\tau, \sigma$ and $T_{c}$ from a given set of $\left\langle n_{a}\right\rangle$ (either from experiment or models) when only an approximate fit is expected is non-trivial and not unique. We skip the details here which are given in $[28,29,30]$. A more sophisticated method of extraction of the relevant parameters can be found in [31]. The same technique is used in [32].

The EOS collaboration [33] obtained data from break up of $1.0 \mathrm{GeV}$ per nucleon gold nuclei on a carbon target. Depending upon the impact parameter, the excitation energy (or the temperature) of the projectile like fragment which breaks up into many composites will vary. In $[28,29]$ it is argued that $T$ in eq.(15) varies linearly with the charged multiplicity $m$ and the scaling function of eq. (15) is changed from $f\left(a^{\sigma}\left(T-T_{c}\right)\right)$ to $f\left(a^{\sigma}\left(\frac{m-m_{c}}{m_{c}}\right)\right)$. Here $m_{c}$ is the critical multiplicity. Having determined from the data $\tau, \sigma$ and $m_{c}$ (as mentioned before we are skipping the details of how the extraction is done but this can be found in $[28,29])$ one then verifies if the scaling law works: that is, we check if for all $a$ 's, $\left\langle n_{a}\right\rangle a^{\tau}$ will fall on the same curve when plotted as a function of $a^{\sigma}\left(m-m_{c}\right) / m_{c}$. How well this works can be seen, for example, in fig.(18) of [30]. The deviations from the hypothetical "universal" curve are by no means negligible but can we assume that the scatter of points is entirely finite particle number effect and conclude that we have indeed seen evidence of critical phenomena?

To resolve this, we play a theoretical game. We take the thermodynamic model (which we know has only a first order phase transition), pick a system with particle number $A$, generate $\left\langle n_{a}\right\rangle$ for different temperatures $T$ and from these data extract best possible values of $\tau, \sigma$ and $T_{c}$. Having obtained these we examine how well the scaling law applies. This is shown in fig. 3 . The figure is taken from [32] where other similar examples are displayed. For the model, deviations from one "universal" curve are smaller than what the EOS collab- 
oration data gave. We might conclude we have extracted the model critical temperature and the critical exponents. These would be wrong conclusions of course because the model has only a first order phase transition. In fact the value of $T_{c}$ one extracts this way is quite close to $T_{b}$, the first order phase transition temperature.

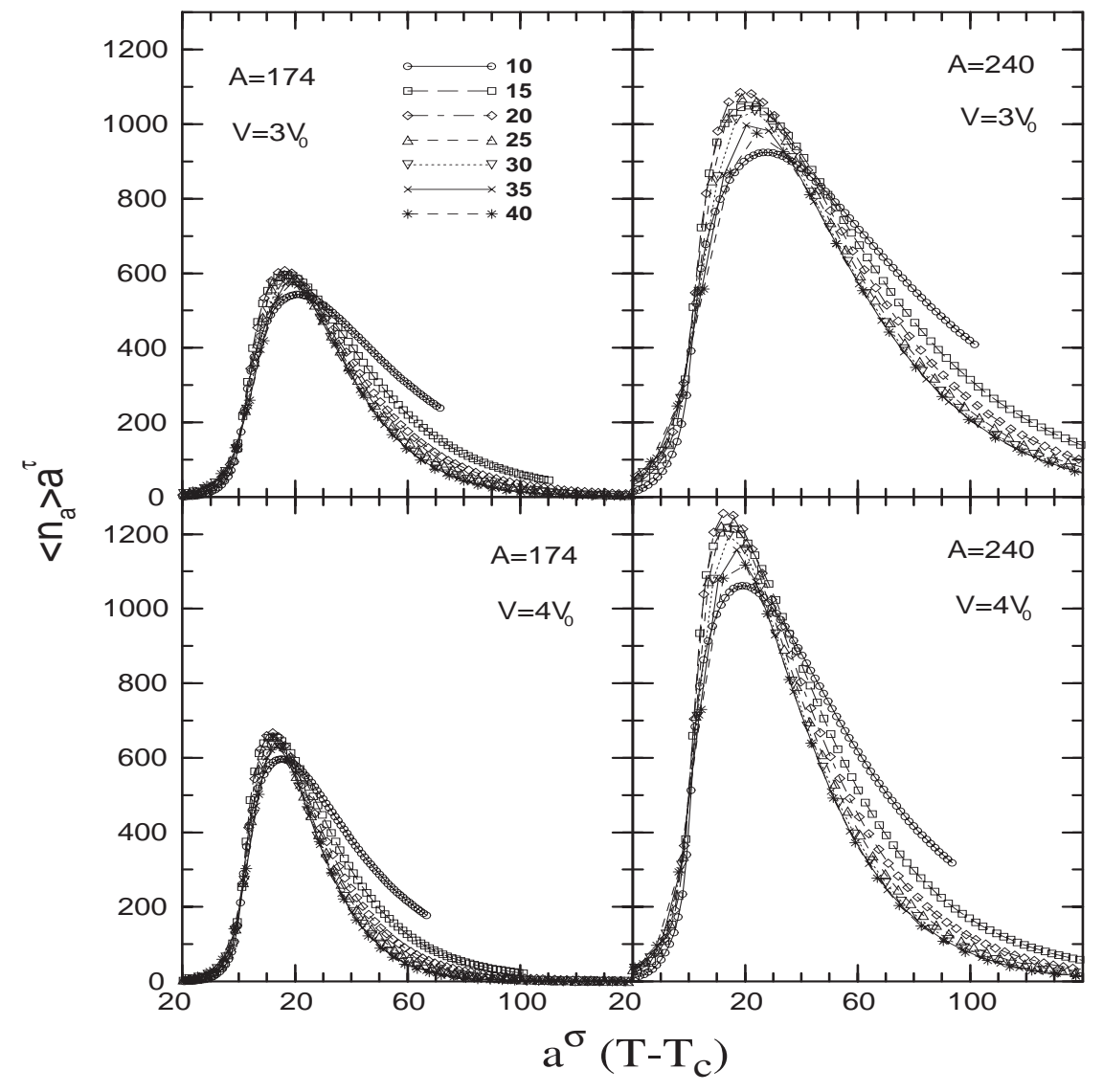

Fig.3. The scaling behaviour in the mass range $10 \leq a \leq 40$ in the thermodynamic model for different systems at different freeze-out densities.

In [30] the Copenhagen SMM is used to show that approximate scaling is obtained. The hope would then be that the theory also demonstrated criticality. The SMM is, in spirit, very close to the thermodynamic model, thus we doubt that the very approximate collapse of $\left\langle n_{a}\right\rangle a^{\tau}$ on one curve is any indication of criticality. It is impossible to disentangle what errors arise because the wrong formula is applied and what errors arise because of the finiteness of the system and many other factors such as the coulomb force, pre-equilibrium emission etc. Experimental data would have a very hard time of choosing between a first and a second order transition. 
From $8 \mathrm{GeV} / \mathrm{c} \pi^{-}$on Au data, the ISiS [34] collaboration obtained the caloric curve [35]. The specific heat was obtained by differentiating with respect to $T$. Experiment shows that the peak of the specific heat coincides well with the position where the $\chi^{2}$ for $\sum\left(\left\langle n_{a}\right\rangle-C a^{-\tau}\right)^{2}$ minimises. Here both $C$ and $\tau$ are taken as parameters to be fixed by minimisation. The canonical model gives similar results. Further details of experiment and theory can be found in [36] where effects of the coulomb interaction on the position of the maximum of the specific heat is discussed in detail.

We turn now briefly to another phenomenological model which was invoked twenty years ago [37] but was revived recently [38]. This is yet another example where evidence for criticality can be drawn too hastily. Consider the formation of a droplet containing $a$ particles in the liquid phase surrounded by $b$ particles in the gas phase. At constant temperature and pressure the Gibbs free energy is the relevant factor. Then

$G_{\text {withdrop }}=\mu_{l} a+\mu_{g} b+4 \pi R^{2} \sigma+T \tau \ln a$

and $G_{\text {nodrop }}=\mu_{g}(a+b)$

The probability of formation of droplet containing $a$ particles is proportional to $\exp (-\Delta G / T)$ so that the yield of droplets of size $a$ is

$$
\left\langle n_{a}\right\rangle=C a^{-\tau} \exp \left[\left(\mu_{g}-\mu_{l}\right) a / T+c_{2} a^{2 / 3} / T\right]
$$

Here both $\mu_{l}$ and $\mu_{g}$ are functions of $T$. At coexistence and also at critical temperature, they become equal to one another. Also $c_{2}$ is a function of temperature and at $T_{c}$, the coefficient $c_{2}$ goes to zero. Since above $T_{c}$, there is no distinction between the liquid and the gas phase, one can not speak of droplets. Thus the theory only applies to $T<T_{c}$. As such, the formulation is more limited than that of eq.(15) which applies to both sides of $T_{c}$. We now generate values of $\left\langle n_{a}\right\rangle$ from the thermodynamic model for different temperatures and try to fit these "data" using eq.(16). The following fit was tried. We set $\tau=2$. Let $\alpha=\left(\mu_{g}-\mu_{l}\right) / T, \gamma=c_{2} / T$. We fit the calculated $\left\langle n_{a}\right\rangle$ to $C a^{-2} \exp \left(\alpha a+\gamma a^{2 / 3}\right)$ at different temperatures where $\alpha, \gamma$ values at each temperature are varied for best fit. The values of $\alpha, \gamma$ as functions of temperature are shown in fig. 4 where we also show that the parametrisation fits the values of $\left\langle n_{a}\right\rangle$ very accurately. The values of $\alpha$ and $\gamma$ both go to zero near temperature $T=6.5 \mathrm{MeV}$ suggesting the critical temperature is $6.5 \mathrm{MeV}$. Of course this conclusion would again be wrong since the model which gave these $\left\langle n_{a}\right\rangle$ 's has only first order phase transition.

One problem is that whenever a fit, whether through eq.(15) or through eq.(16), is done, the fit is attempted for a narrow range, $a=6$ to 40 . In this limited range moderate to excellent fits are obtainable for different looking 
parametrisations. It is shown in [32] that if the range of $a$ could be extended to beyond 100, different parametrisations would diverge. Unfortunately, the range of $a$ has to be limited. For example higher values of $a$ would have contamination from fission processes which is something we do not wish to include. If we are stuck to a limited range of $a$ 's we will also be limited by ambiguity.
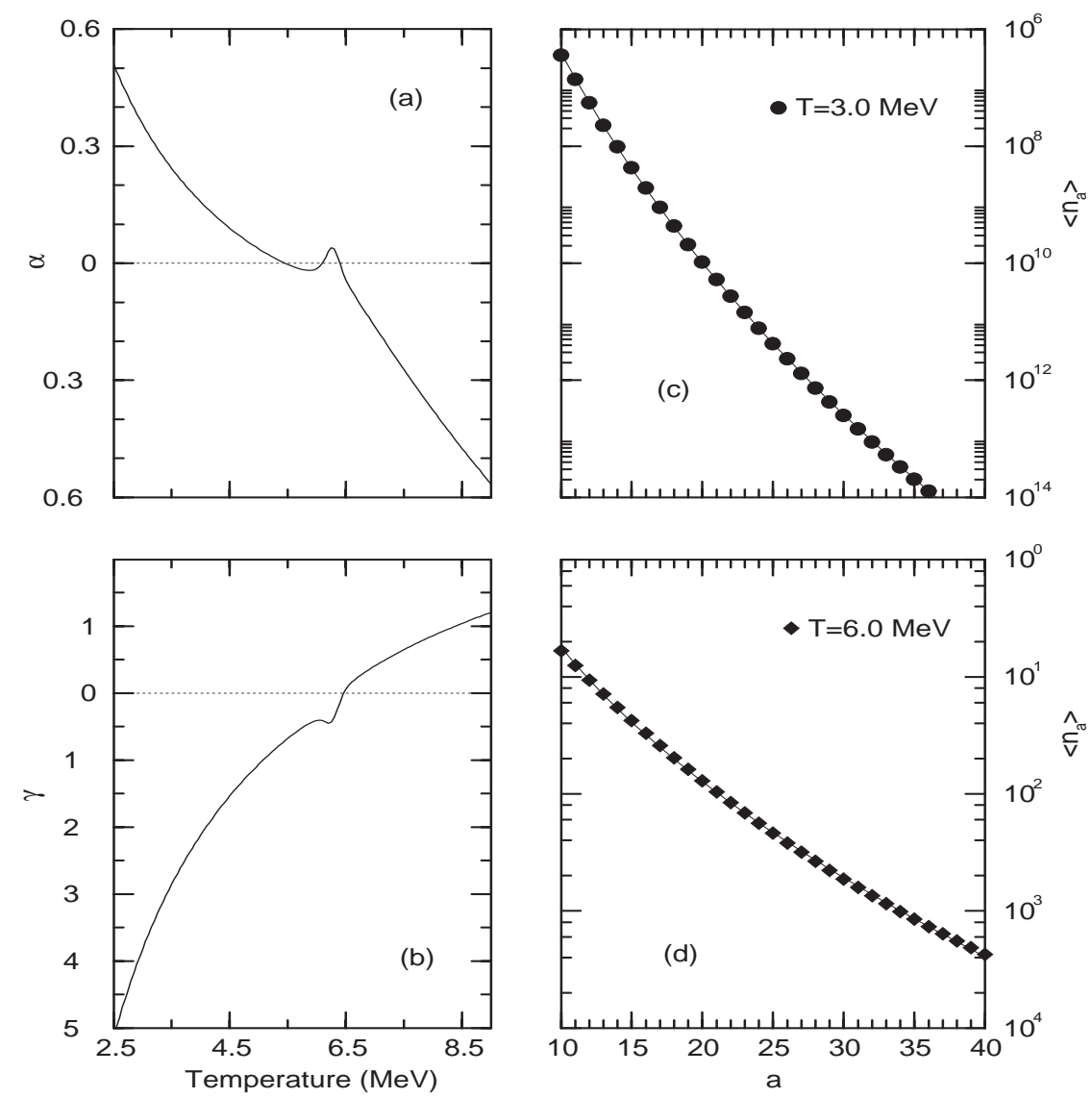

Fig.4. The parameters of the droplet model $\alpha$ and $\gamma$ as a function of temperature for a system of 240 particles at freeze-out volume $4 V_{0}$. The right panels show the fit of the model to the yields obtained in the thermodynamic model

The emphasis towards unravelling critical phenomena from data on intermediate energy heavy ion collisions is at least partly due to history. The observation by the Purdue group [39] that the yields of the fragments produced in $p+X e$ and $p+K r$ obeyed a power law $\left\langle n_{a}\right\rangle \propto a^{-\tau}$ led to a conjecture that the fragmenting target was near the critical point of liquid-gas phase transition. The origin of this conjecture is the Fisher model [27] which predicts that at the critical point the yields of the droplets will be given by a power law. Also the first microscopic model that was used $[19,20]$ to compute yields of fragments was the percolation model which has only a continuous phase transition and a 
power law at criticality. The power law is no longer taken as a "proof" of criticality. There are many systems which exhibit a power law: mass distribution of asteroids in the solar system, debris from the crushing of basalt pellets [40] and the fragmentation of frozen potatoes [41]. In fact the lattice gas model which has been used a great deal for multifragmentation in nuclei gives a power law at the critical point, at the co-existence curve (this is a first order phase transition provided the freeze-out density is less than half the normal density) and also along a line in the $T-\rho$ plane away from the coexistence curve $[42,43,44]$.

We finish this section stating that the lattice gas model which has a Hamiltonian and can be and has been used to fit many data (not in any obvious way connected with phase transition) also predicts a first order phase transition at intermediate energy $[42,43]$.

\subsection{Comparision with mean-field theory}

Here we concentrate on the thermodynamic model but as applied to nuclei with neutrons and protons. The operative equations are (10) to (13) but we will switch off the coulomb term ( $\kappa$ of eq.13 will be set to zero). The objective is to compare with finite temperature Hartee-Fock results for nuclear matter. For nuclear matter the coulomb interaction has to be switched off and one retains only the nuclear part of the interaction.

Phase transitions are often considered in the mean-field model. Examples for the present discussion are $[10,12,13]$. Invariably a grand canonical ensemble is used characterised by a neutron chemical potential $\mu_{N}(T, \rho)$ and a proton chemical potential $\mu_{P}(T, \rho)$. The use of the grand canonical model would imply that the results are valid for very large systems although in nuclear physics we often use the grand canonical ensemble for not so large systems as well.

Muller and Serot $[45,46]$ used the mean-field model to investigate phase transition in nuclear matter. Normally nuclear matter means a very large system with $N=Z$ with the coulomb force switched off. For this section we will use the term nuclear matter for very large systems but $N$ can be different from $Z$. The coulomb is switched off as usual. Define proton fraction $y=Z /(N+Z)$. Symmetric nuclear matter has $y=0.5$ and would have a first order phase transition below the critical point. But for $y$ deviating significantly from 0.5 , these authors demonstrate with a more general Maxwell like construction that the first order phase transition would turn into second order. Further the phase transition would take place neither at constant volume nor at constant pressure but would have a more general path to traverse.

The general characteristics of mean-field theories is that one is constrained to 
have one density. Having the same density everywhere is a big price to pay. For example, this would not permit a liquid phase at one place and a gas phase at another. The limitation of one density only shows up as mechanical instability, i.e., in parts of the equation of state diagram ( $p-\rho$ isothermals) $\partial p / \partial \rho$ turns out to be negative. This is unacceptable for infinite matter and then one has to, by hand, correct this using a Maxwell construction. The thermdynamic model is very different. Here, for example, $\rho / \rho_{0}=0.3$ does not mean that at the freeze-out volume, matter is uniformly stretched. Rather matter breaks up into different blobs all with the same density $\rho_{0}$ but there are empty spaces between blobs. If there is a large blob, we identify it as liquid, nucleons and light composites in the adjoining spaces form the gas (In [47], it is shown that this last scenario has a lower free energy compared to uniform stretching as asumed in Hartree-Fock theory). For large matter, there is no need for $(\partial p / \partial \rho)_{T}$ to be negative.

A similar thing happens with isospin fractionation. In mean-field theory, there is one value of $y$ everywhere. Experimentally, it is verified that if the dissociating system has a small $y$, then after break up, the largest blob has $y>y_{\text {diss }}$ whereas $n_{p} /\left(n_{p}+n_{n}\right)<y_{\text {diss }}$ where $n_{p}, n_{n}$ are free protons and free neutrons respectively. Here $y_{\text {diss }}$ is the the $y$ value of the dissociating system. One might say the liquid phase has a different $y$ from that of the gas phase. Again meanfield theory would have a hard time accommodating this. It must have the same value of $y$ everywhere and the fact that this is an unstable situation shows up in the following way. If we draw $\mu_{P}\left(\mu_{N}\right)$ as a function of $y$ at constant temperature, the derivative $\left(\partial \mu_{P} / \partial y\right)_{p}$ can turn out to be negative (equivalently $\left(\partial \mu_{N} / \partial y\right)_{p}$ can turn out to be positive). In the thermodynamic model, isospin fractionation happens naturally. In general, the model has, as final products, all allowed composites, $a, b, c, d \ldots$, where the composite $a$ has $y_{a}=i_{a} /\left(i_{a}+j_{a}\right)$ where $i_{a}, j_{a}$ are the proton and neutron numbers of the composite $a$. The only law of conservation is $Z=\sum_{a} i_{a} \times n_{a}$ and $N=\sum_{a} j_{a} \times n_{a}$. So a large chunk can exist with higher $y$ than that of the whole system and populations of other species can adjust to obey overall conservation laws. Whatever partition lowers the free energy will happen. Since we are using a canonical model, we do not need the chemical potentials $\mu_{P}$ or $\mu_{N}$ but we can compute them anyway from the relation $\mu=(\partial F / \partial n)_{V, T}$. We know the values of $Q_{Z, N}, Q_{Z-1, N}$ and $Q_{Z, N-1}$. Since $F=-T \ln Q$, one has $\mu_{P}=-T\left(\ln Q_{Z, N}-\ln Q_{Z-1, N}\right)$ and similarly for $\mu_{N}$.

Calculations with the canonical model discussed in this article, do not show regions of negative $\left(\partial \mu_{P} / \partial y\right)_{p, T}[48,49]$. These also suggest that the phase transition in the canonical model remains first order for asymmetric matter. We show in fig.5 results of $c_{V}$ calculation for different degrees of asymmetry. One sees that as the system gets bigger, the maximum in $c_{V}$ becomes narrower and higher, ensuring there will be a break in the first derivative of the free energy in the large matter limit. 
Chemical instability for finite systems in Hartee-Fock theory has also been worked out. Contributions of both coulomb and surface terms can be included. For details see $[51,52,53]$.

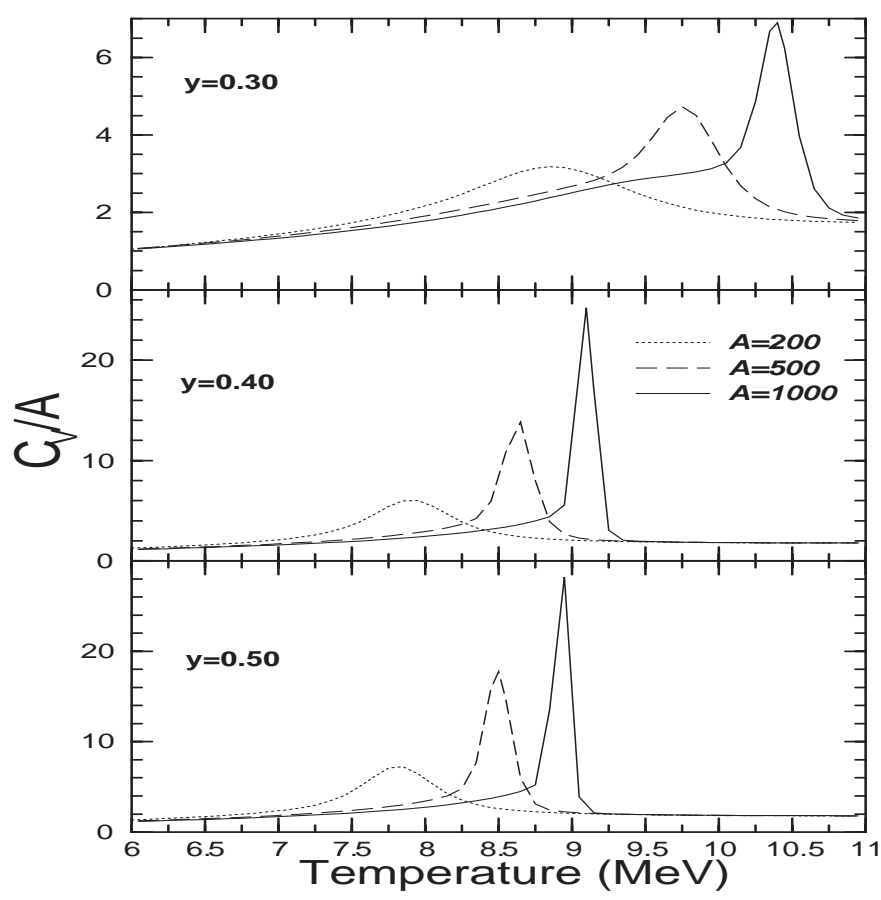

Fig.5. The $C_{V} / A$ as a function of temperature for systems of 200,500 and 1000 particles with different proton fractions: $(y=Z / A)$.

\section{Comparison of canonical and grand canonical}

As noted in the introduction, the grand canonical version of the model we are pursuing in this paper has been known and used for a long time. Now that we know how to treat an exact number of particles rather than an ensemble of particle numbers, it will be useful in a few cases to examine, given that our dissociating system has an exact number of particles, how the use of grand canonical ensemble affects the prediction of observables. For simplicity, we start with the model of one kind of particles and our dissociating system has 200 particles. Thus we can have monomers, dimers, trimers,...upto a composite of 200 particles. In the grand canonical ensemble, the average number of composites with $k$ nucleons is given by

$$
\left\langle n_{k}\right\rangle=\exp (\beta \mu k) \omega_{k}=\exp (\beta \mu k) V \tilde{\omega}_{k}
$$


Here $\beta$ is the inverse of temperature and $\omega_{k}$ is the same as defined in eq.6 and $\mu$ is the chemical potential. We also use $\tilde{\omega}=\omega / V$ where $\tilde{\omega}$ only depends upon the composite and the temperature but not upon the volume of the dissociating system. The chemical potential is determined by solving

$$
\rho=\sum_{k=1}^{k_{m}} k \exp (k \beta \mu) \tilde{\omega}_{k}
$$

In this example $k_{m}=$ the number of particles in the largest cluster $=200$. Having determined $\mu$ we now find $\left\langle n_{k}\right\rangle$ from $\left\langle n_{k}\right\rangle=\exp (k \beta \mu) V \tilde{\omega}_{k}$.
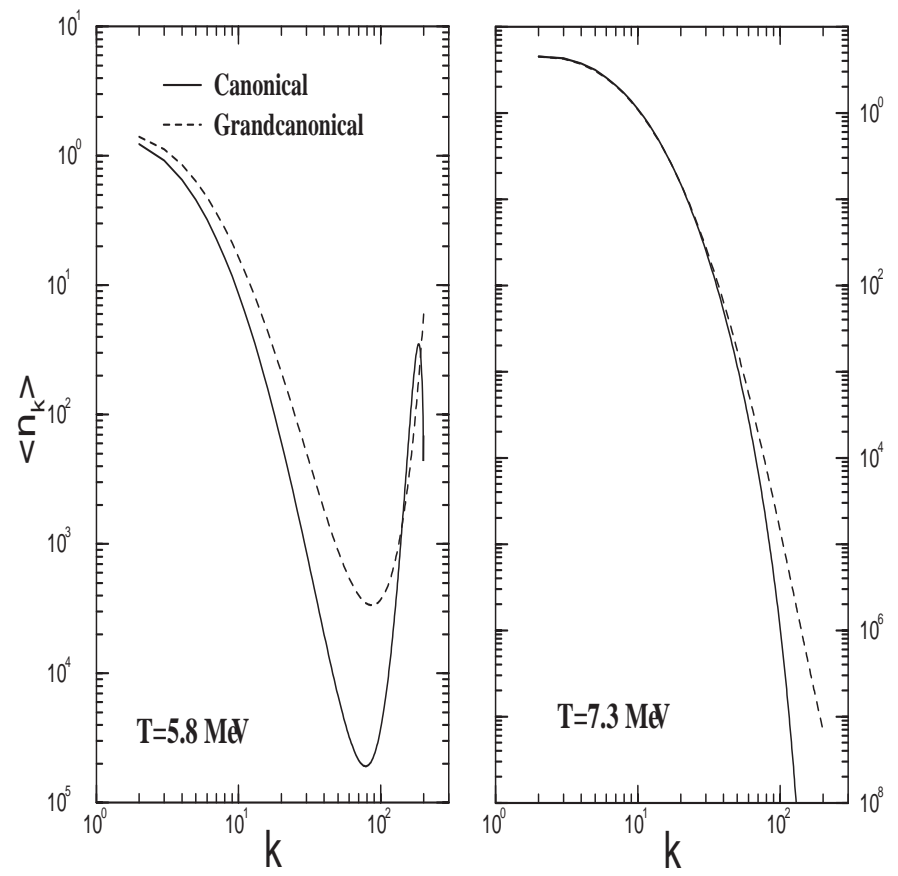

Fig.6. Comparison of yields obtained in the canonical and grand canonical models at different temperatures, for a system of 200 particles at freeze-out volume $3 V_{0}$.

In figs. 6 and 7 , we make a comparison of $\left\langle n_{k}\right\rangle$ 's from canonical and grand canonical ensembles. The value of $V$ was set at $3 V_{0}$. Results are shown for temperatures below the phase transition temperature and above it. Fig. 6 seems very reasonable. The overall features are similar. The differences get highlighted in fig. 7. At temperature $7.3 \mathrm{MeV},\left\langle n_{k}\right\rangle^{G C}$ and $\left\langle n_{k}\right\rangle^{C}$ are practically the same upto $k=40$ but deviate wildly afterwards. Since most of the time we are not interested in the heavier products and $k=40$ is the limit of intermediate mass fragments one is investigating, the grand canonical ensemble does an 
adequate job. We must be aware however, that, for heavy composites the grand canonical ensemble does a very poor job. The accuracy of the grand canonical ensemble at temperature $5.8 \mathrm{MeV}$ (below the phase transition temperature) is absolutely awful for almost all composites. This is also the temperature range appropriate for most intermediate energy reactions. It is thus dangerous to use the grand canonical ensemble in intermediate energy heavy ion reactions.

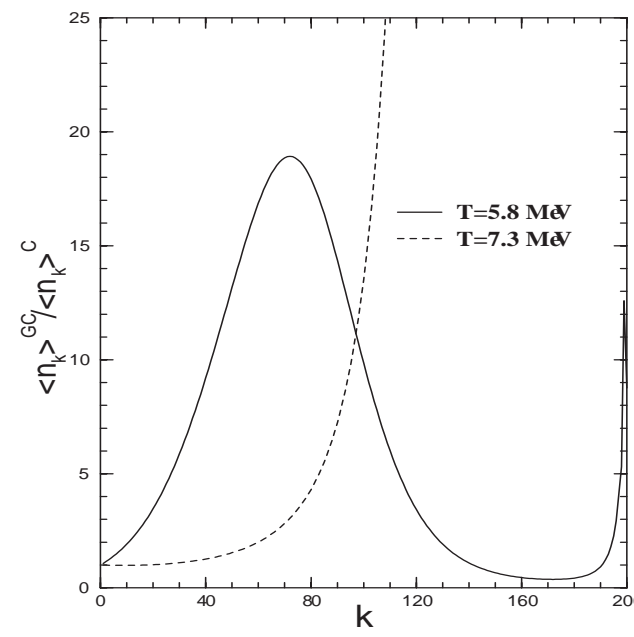

Fig.7 The ratio of yields obtained in the grandcanonical and the canonical model at different temperatures.

If however, one is only interested in finding the ratio of populations of two adjacent composites, the grand canonical continues to be useful over a larger domain. This is shown in fig.8.
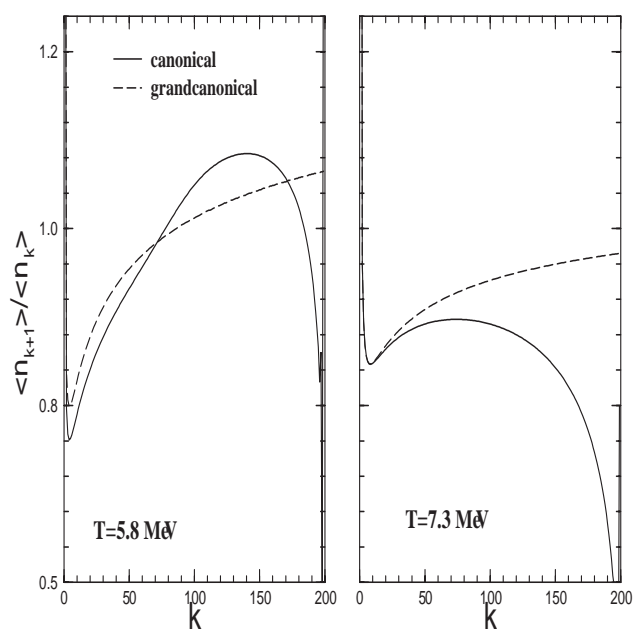

Fig.8 Ratio of yields of adjacent composites in the two models. 
The very different populations of composites below the phase transition temperature leads to drastically different caloric curves in the grand canonical ensemble and canonical ensemble. As noted in section 4 and shown in fig.2, for a fixed density the specific heat per particle maximises at a certain temperature. Keeping density fixed, if we increase the number of particles the height of the maximum increases and the width decreases. In fig.9 we show this again for 200 and 2000 particles, but now we have also indicated the specific heat calculated in the grand canonical ensemble. In both the models, the peak of the specific heat increases when we go from 200 to 2000 particles and the widths decrease but the results are much more dramatic in the canonical model. In particular, it is not obvious that the specific heat in the grand canonical ensemble will attain extraordinary heights and miniscule widths. In fact, it was suggested in the literature, engaging the grand canonical ensemble, that there is a discontinuity in the value of the specific heat at phase transition but no infinity [23].

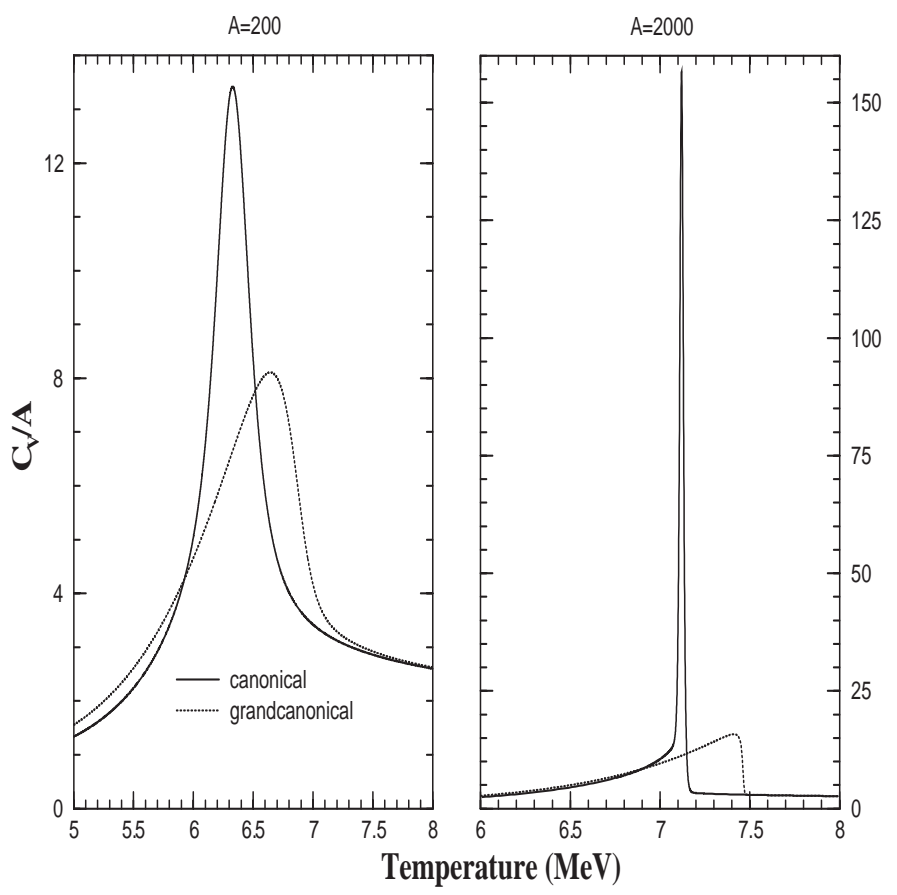

Fig.9 Specific heat per particle at constant volume when the system has total number of particles 200 and 2000. Canonical and grand canonical values are shown.

To understand at a more fundamental level the cause of the difference in values of specific heats in the two ensembles, we will analyse the case of 2000 particles in some detail. In the grand canonical model, even though we are using the average value of the particle number to be 2000 , there are, in practice, systems 
with varying particle numbers (in principle, from 0 to $\infty$ ). The part which has, for example, 1000 particles has density half of the prescribed density. The peak in the specific heat of this half density will occur at a different temperature than that which maximises the specific heat at density 2000/V. Thus there is a smearing effect. This is always an inherent problem with using the grand canonical ensemble but most of the time the fluctuation from the average value is small enough that one can live with it. This would have meant, in our present example, the part which contains 1000 particles is so negligibly small that it does not matter. This however is not so in the present model below the phase transition temperature.

In the present case, the grand canonical calculation starts out by obtaining $\mu$ from eq.18 where $k_{m}=2000 ; \rho$ was taken to be $\rho_{0} / 2.7$. The average value of $\left\langle n_{k}\right\rangle$ is then given by eq.(17) where $V=2000 \times 2.7 / \rho_{0}$. With this we have $\sum_{k=1}^{k=2000} k\left\langle n_{k}\right\rangle=2000$. The fluctuations in the model can be calculated easily. We have the general statistical relation

$$
\frac{1}{\beta^{2}} \frac{\partial^{2} \ln Q_{\text {gr.can }}}{\partial^{2} \mu}=\left\langle N^{2}\right\rangle-\langle N\rangle^{2}
$$

Here $Q_{\text {gr.can }}$ is the grand canonical partition function. We can write two expressions for $Q_{\text {gr.can. }}$. One is:

$$
\ln Q_{\text {gr.can }}=\sum_{k=1}^{k=2000} \exp (\beta \mu k) \omega_{k}
$$

This immediately leads to

$$
\left\langle N^{2}\right\rangle-\langle N\rangle^{2}=\sum_{k=1}^{k=2000} k^{2}\left\langle n_{k}\right\rangle
$$

which is easily calculable. The other expression we can exploit in the present case is

$$
Q_{\text {gr.can }}=\sum_{K=1}^{\infty} \exp (\beta \mu K) Q_{K, k_{m}}
$$

where $Q_{K, k_{m}}$ is the canonical partition function of $K$ nucleons but with the restriction that the largest cluster can not have more than $k_{m}(=2000)$ nucleons. We can calculate these explicitly using methods of section 2 . For practical reasons, $K$ has to be cut off at the upper end. Here we used $K=10000$ as the upper limit. Since the average number of particles is 2000, this appears to be a safe upper limit in eq. 22. The quantity $\mu$ is known from solving eq. 18. 
The fluctuations calculated with eqs. 21 and 22 are shown in fig. 10. One sees there is a temperature above which the fluctuations are small. At these temperatures, the grand canonical value of specific heat is indistinguishable from the canonical value. But as the temperature is lowered, fluctuations grow rapidly and the results begin to diverge.

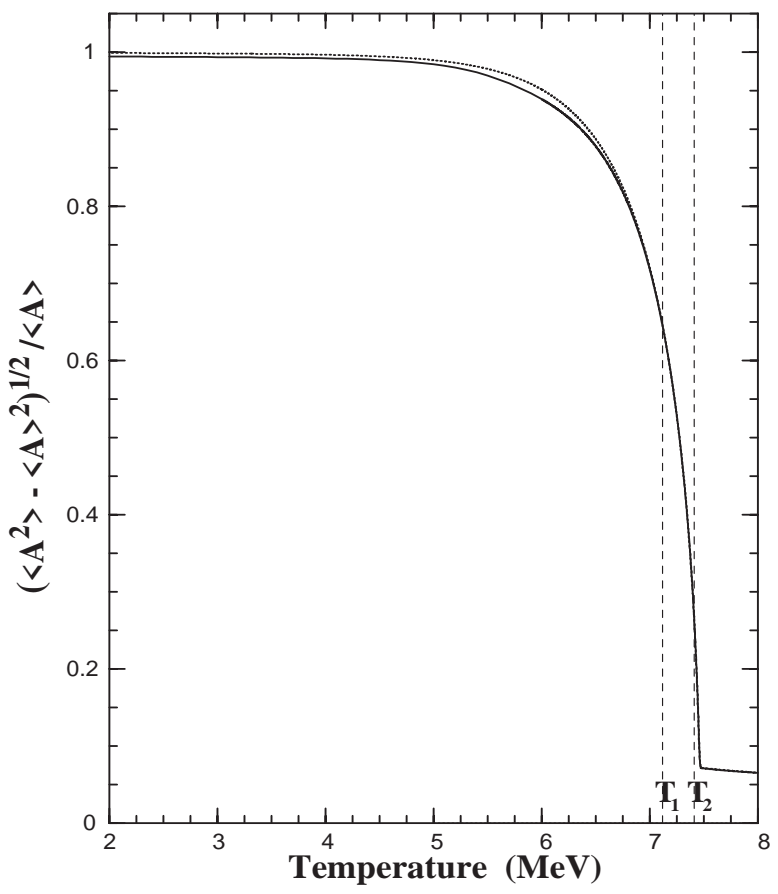

Fig.10 Fluctuations calculated using eqs. (21) and (22). The solid line corresponds to using eq. (22) with $K$ cut off at 10,000 and the dotted line corresponds to using eq. (21). $T_{1}$ corresponds to to the temperature where the specific heat maximises in the canonical calculation and $T_{2}$ to the temperature of highest specific heat in the grand canonical calculation.

It is interesting to study fluctuations further. The probability of $K$ particles being in the grand canonical ensemble is $\propto e^{K \beta \mu+l n Q_{K}}$. We plot in fig.11 $\exp \left[\beta \mu(K-A)+\ln Q_{K}-\ln Q_{A}\right]$. This takes the value 1 at $K=A$ and in the normal picture of the grand canonical ensemble would drop off rapidly on either side of $A$. This does happen at temperatures higher than the boiling temperature. The case at $T=7.7 \mathrm{MeV}$ corresponds to a standard scenario. But the situation at temperature $7.3 \mathrm{MeV}$ is drastically different. The probability does not maximise at $K=A$ but at a lower value. It is also very spread out with a periodic structure. The periodicity is 2000 and is linked with the fact that in the case studied, the largest composite has 2000 nucleons and at low temperatures, this composite will play a significant role.

More discussions on this case can be found in [50]. 

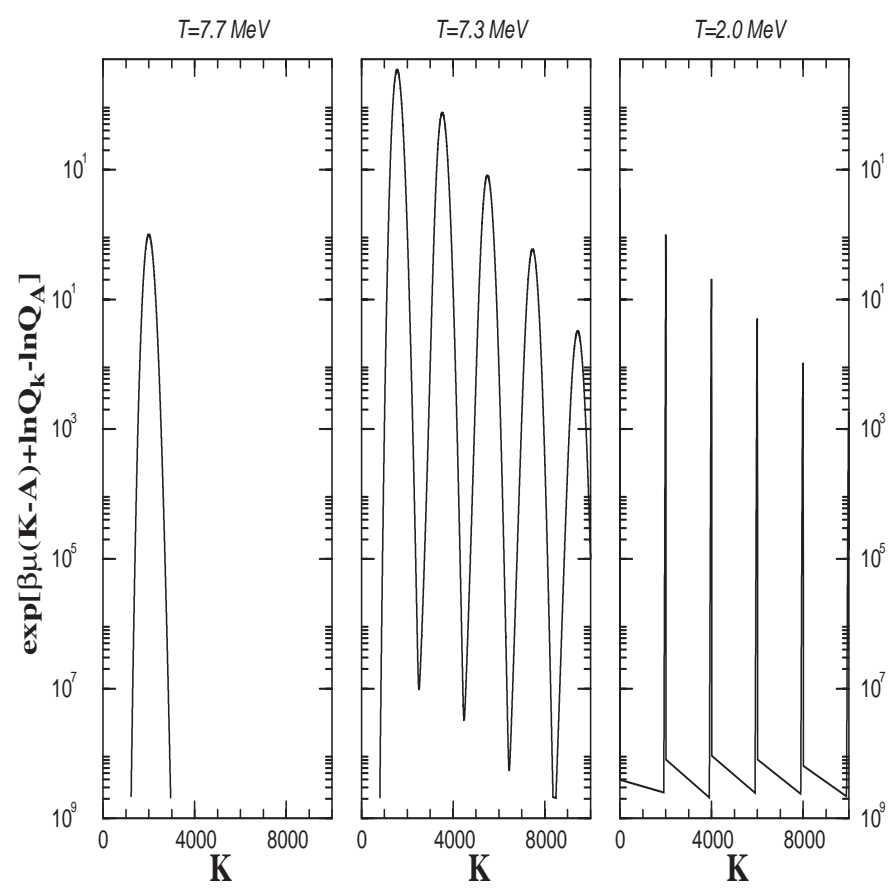

Fig. 11. These graphs show the spread of particle numbers in the grand canonical ensemble when the average particle number is 2000. The spread is very narrow at temperature $7.7 \mathrm{MeV}$ but becomes quite large at lower temperatures.

\section{Specific heat at constant pressure}

We have used $C_{V}$, the specific heat at constant volume a great deal in the previous sections. In canonical models $C_{V}$ is always positive. Writing: $\langle E\rangle=$ $\frac{\sum E_{i}(V) \exp \left(-\beta E_{i}(V)\right)}{\sum \exp \left(\left(-\beta E_{i}(V)\right)\right.}$ and $C_{V}=(\partial\langle E\rangle / \partial T)_{V}$, we get $C_{V}=\frac{1}{T^{2}}\left\langle(E-\langle E\rangle)^{2}\right\rangle$ which is the expectation value of a positive definite operator. However, specific heat at constant pressure allows no such generalisations. Here we enter into a discussion of the specific heat at constant pressure in the thermodynamic model. We should add that dissociation after two heavy ions collide is largely an uncontrollable situation and we do not know what is a better description: disassembly at constant volume, disassembly at constant pressure or a hybrid situation.

Lately, interest in the topic has increased with the realisation that for finite systems, $C_{p}$ can sometimes be negative and such cases might arise in heavy ion collisions $[54,55,56]$. To study this possibility in our model, we find it convenient to look at the $p-\rho$ diagram at constant temperatures (isothermals). 
This is shown in fig.12. We see there are regions of mechanical instability where $\left(\frac{\partial p}{\partial \rho}\right)_{T}<0$. We will show that the occurrence of negative $C_{p}$ happens in this region.

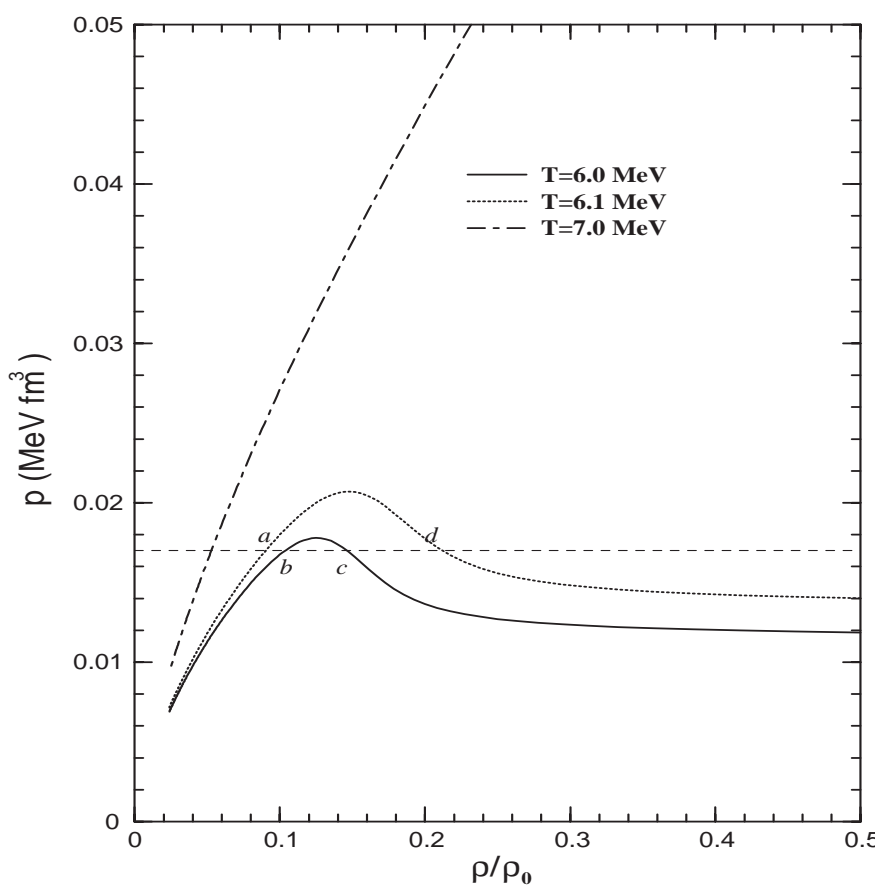

Fig. 12. EOS in the canonical model for a system of $A=200$ particles. The largest cluster also has $N=200$.

The most famous case of mechanical instability is the Van der Waals equation of state. In nuclear physics, if one uses the Hartree-Fock theory, then also large regions of mechanical instability appear. Examples of this can be seen in many published works: $[10,9,48]$. All these published works are for infinite systems (unlike the $p-\rho$ diagram for fig. 12 which is drawn for 200 particles). Quantitative examination of the equation of state diagrams reveal that the regions of mechanical instability are far bigger in the case of Hartree-Fock as opposed to what we see in fig.12. In fact, plotted on the same scale, the region of mechanical instability would be tiny (ref. fig.1 of [48]) and one would have to plot it in an expanded scale (such as is done in fig.12) to study it quantitatively.

In the Van der Waals case or in the Hartree-Fock case for infinite nuclear matter one uses a Maxwell construction to replace the region of mechanical instability [2]. In the thermodynamic limit, regions of mechanical instability should disappear. In our case there is no prescription for Maxwell construction. Also since our system is very finite, we take the mechanical instability in fig.12 as real and follow the consequences for the specific heat. In the figure we have 
drawn isothermals at three temperatures; $T_{1}<T_{2}<T_{3}$. Here $T_{2}$ is only slightly higher than $T_{1}$. Instead of $\rho$ let us use the variable $V \propto 1 / \rho$. The pressure is given by $p=T(m / V)$ where $m$ is the multiplicity. [We actually use $m-1$ but this is inconsequential for the discussion to follow.] For the simple case of monomers only, $p$ is given by $p=T(A / V)$ where $A$ is the number of particles. This number does not change thus $p$ keeps falling with $V$. In our case, $m$ is significantly less than $A$. It is not a constant as $V$ and/or $T$ change. As can be readily guessed, $m$ increases with $T$ at constant $V ; m$ also increases with $V$ at constant $T$. Negative compressibility is marked by $(\partial m / \partial V)_{T}>m / V$.

Let us consider the points $c$ and $d$ in fig. 12. Let $c$ have multiplicity $m$, volume $V$ and temperature $T$; for $d$ the corresponding quantities are $m+\delta m, V+\delta V$ and $T+\delta T$. Here $\delta V$ is negative, $\delta T$ is positive. Using

$$
p=T \frac{m}{V}=(T+\delta T) \frac{m+\delta m}{V+\delta V}
$$

we arrive at

$$
\frac{\delta m}{m}=\frac{\delta V}{V}-\frac{\delta T}{T}
$$

In the region $(c, d), \delta V$ is negative, $\delta T$ is positive thus $\delta m$ is negative. If $m$ goes down then so does the potential energy (creating more $m$ creates more surface and hence increases energy). The change in kinetic energy is : $\frac{3}{2}[(m+\delta m)(T+\delta T)-m T]$ which using eq.24 is $\approx \frac{3}{2} \frac{\delta V}{V} m T$. This is negative also. Thus both kinetic and potential energy fall giving rise to negative $C_{p}$. If on the other hand we consider points $a$ and $b$, point $a$ has both a bigger volume and a bigger temperature thus $\delta m$ is positive. This would make both the kinetic and potential energy rise when one moves from $b$ to $a$. This is illustrated in Table 1. The caloric curve of fig. 13 shows regions of negative $C_{p}$. 
Table 1

Variation of energies per particle $(\mathrm{MeV})$ with temperature $(\mathrm{MeV})$ in the negative and positive compressibility zones, for $p=0.017 \mathrm{MeV} \mathrm{fm}^{-3}$

\begin{tabular}{cccccc}
\hline & $T$ & $\rho / \rho_{0}$ & $e_{k} / A$ & $e_{\text {pot }} / A$ & $e_{\text {tot }} / A$ \\
\hline & 6.0 & 0.146 & 0.978 & -5.235 & -4.257 \\
$\frac{\partial p}{\partial \rho}<0$ & 6.1 & 0.212 & 0.638 & -6.970 & -6.332 \\
& 6.2 & 0.392 & 0.294 & -8.708 & -8.414 \\
\hline \multirow{3}{*}{$\frac{\partial p}{\partial \rho}>0$} & 6.1 & 0.090 & 1.653 & -2.513 & -0.859 \\
& 6.2 & 0.082 & 1.824 & -2.027 & -0.202 \\
\hline
\end{tabular}

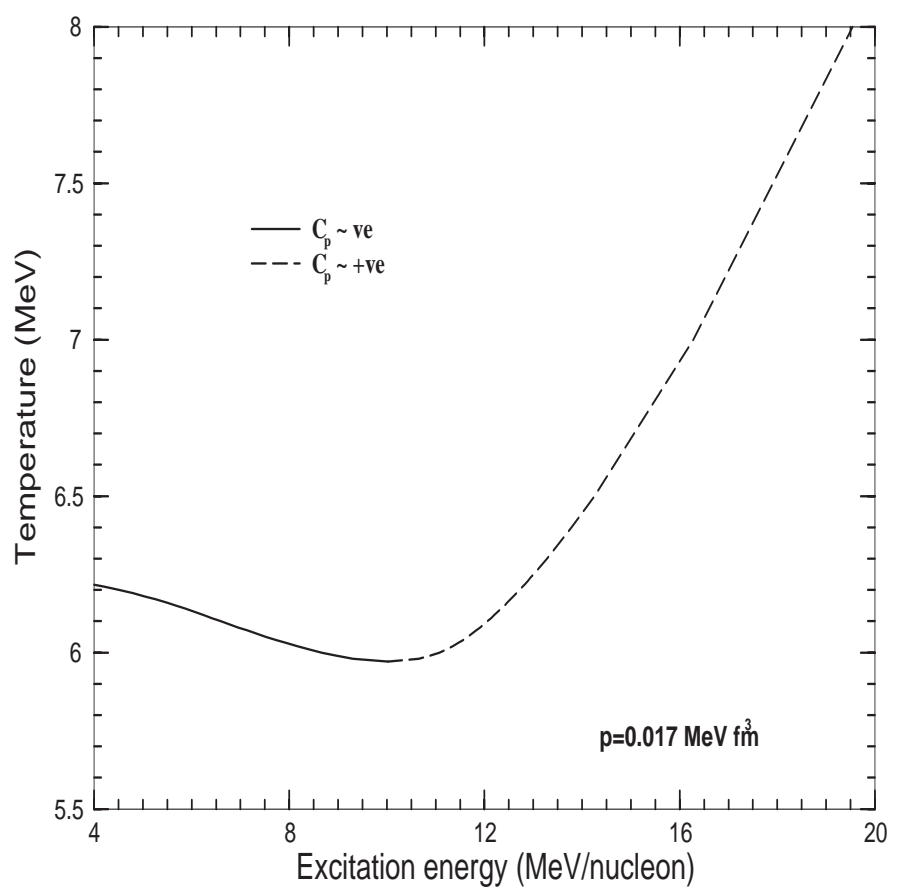

Fig. 13. Caloric curve at constant pressure $\left(p=0.017 \mathrm{MeV} \mathrm{fm}^{-3}\right)$ in the canonical model with $A=200$ and $N=200$. The solid and dashed portions of the curve give -ve and + ve $C_{p}$ respectively.

Let us consider the thermodynamic limit. This will be reached when the number of composites near the boundaries of the freeze-out volume is negligible to the number of composites well inside. In this limit, intensive variables remain unchanged when extensive variables are changed by a constant factor. Thus if $A$ is the total number of nucleons in the system and we change, at constant temperature, $A \rightarrow A+\alpha A, V \rightarrow V+\alpha V$ the pressure $p=\frac{m}{V} T$ must remain constant. This means, for constant $T$, when $A \rightarrow A+\alpha A, V \rightarrow V+\alpha V, m$ must change to $m \rightarrow m+\alpha m$. Now for compressibility, $A$ stays at $A$, but $V$ 
to $V+\alpha V$ thus $m$ must change to less than $m+\alpha m$. Then the pressure will fall when $V$ is increased, i.e., regions of negative compressibility disappear.

It would be nice to demonstrate this feature directly by doing canonical calculations for larger and larger systems. The area over which negative compressibility appears does drop as larger and larger systems are used but the convergence is slow. Instead we will use the grand canonical ensemble to get to the $A=\infty$ limit. For a given density we solve eq.18, setting once $k_{m}=200$ and $k_{m}=2000$, the other time. This means, in the first case, the largest composite has 200 nucleons and in the second case, the largest composite has 2000 nucleons. The temperature is chosen to be $6 \mathrm{MeV}$. Eq.18 has no reference to either $A$ or $V$ (only their ratio), the implication being for the grand canonical model to be good each factor is $\infty$ or very large. Pressure in the grand canonical ensemble is given by $p=(T / V) \ln Q_{\text {grand }}$ which leads to $p=\sum_{k=1}^{k_{m}} \exp (k \mu \beta) \tilde{\omega}_{k}$.

Fig. 14 compares canonical calculation with $A=200$ and $k_{m} \equiv N=200$ with $A=\infty$ and $N=200$. We see in the low density (the gas phase) the two diagrams coincide. The rise of pressure with density is quite rapid and linear. After the two diagrams separate, the rise of pressure with density in the grand canonical ensemble slows down considerably but there is no region of mechanical instability although the canonical calculation with 200 particles has a region of instability. In the grand canonical result which represents the thermodynamic extrapolation, we have not reached the classic liquid-gas coexistence limit where there would not be any rise of pressure at all (such as in Maxwell's construction). We think the reason is this. The largest cluster is 200 which is not a big enough number. We now increase the largest cluster size to 2000. Now the coexistence region is very clear and there is unmistakable signature of first order phase transition. In the same figure we also show results of canonical calculation with $A=2000$ and $N=2000$. The region of mechanical instability has gone down considerably but it has not disappeared showing that we have not reached the thermodynamic limit yet.

Thermodynamics allows $C_{p}$ to become negative. The following well-known relation exists [2]:

$$
C_{p}-C_{V}=V T \frac{\alpha^{2}}{\kappa}
$$

where $\alpha$ is the volume coefficient expansion and $\kappa$ is the isothermal compressibility given by

$$
\alpha=\frac{1}{V}\left(\frac{\partial V}{\partial T}\right)_{p}
$$




$$
\kappa=-\frac{1}{V}\left(\frac{\partial V}{\partial p}\right)_{p}
$$

For negative $\kappa, C_{p}$ is less than $C_{V}$ and can become negative.

Using the equality $\left(\frac{\partial V}{\partial T}\right)_{p}=-\left(\frac{\partial V}{\partial p}\right)_{T}\left(\frac{\partial p}{\partial T}\right)_{V}$ we can also write

$$
C_{p}-C_{V}=T\left(\frac{\partial p}{\partial T}\right)_{V}\left(\frac{\partial V}{\partial T}\right)_{p}
$$

This shows that $C_{p}$ can drop below $C_{V}$ if the isobaric volume coefficient of expansion becomes negative which is the case in some regions of fig. 11 .

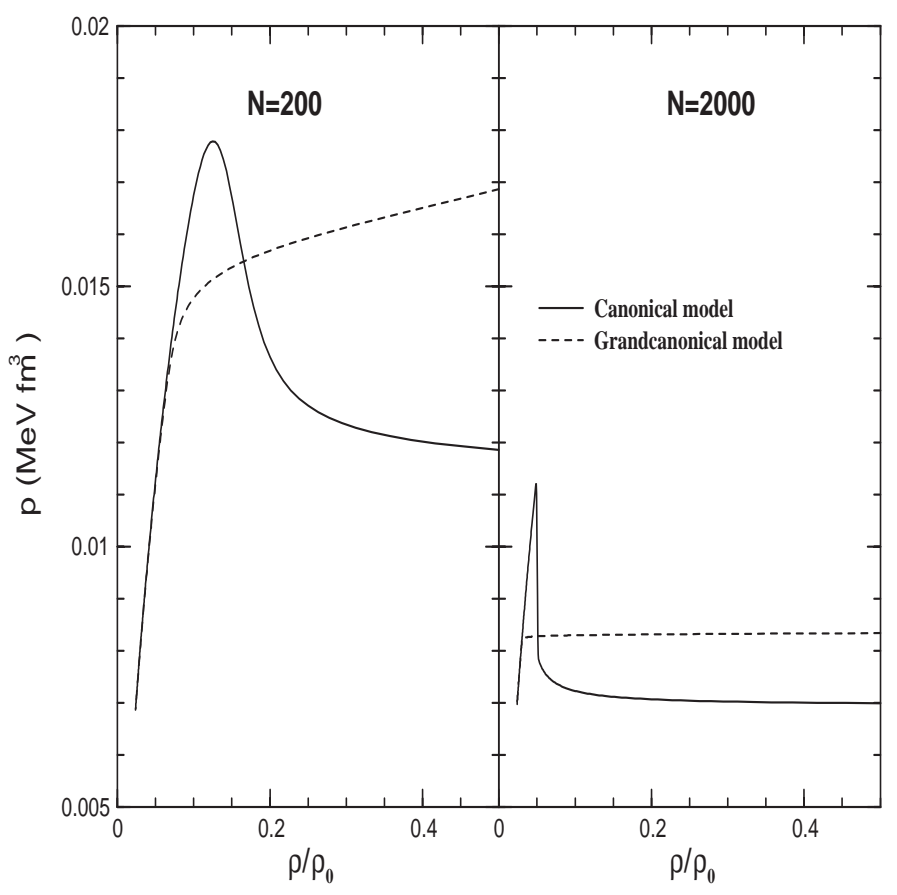

Fig. 14. EOS at $T=6 \mathrm{MeV}$ in the two models. For the left panel, the largest cluster has $N=200$ and for the right panel $N=2000$. For the canonical calculation, the left panel has $A=200$ and the right panel has $A=2000$. For the grand canonical calculation $A=\infty$.

We leave now general considerations of phase transitions, specific heat, caloric curves etc. and explore the predictive powers of the canonical thermodynamic model in producing detailed data in heavy ion reactions. Specifically we will investigate how effective the canonical thermodynamic model is in predicting isotopic yields in some specific reactions. For this we need to go beyond the production of hot fragments that the canonical thermodynamics will give. To obtain yields of specific final products, we need to investigate how fragments at non-zero temperatures will decay. The next sections address this issue. 


\section{Corrections for secondary decay}

The statistical multifragmentation model described above calculates the properties of the collision averaged system that can be approximated by an equilibrium ensemble. Ideally, one would like to measure the properties of excited primary fragments after emission in order to extract information about the collisions and compared directly with the equilibrium predictions of the model described in this report. However, the time scale of a nuclear reaction $\left(10^{-20}\right.$ $\mathrm{s})$ is much shorter than the time scale for particle detection $\left(10^{-9} \mathrm{~s}\right)$. Before reaching the detectors, most particles decay to stable isotopes in their ground states. Thus before any model simulations can be compared to experimental data, it is indispensable to have a model that simulates sequential decays. This turns out to be not a simple task.

In this section, we follow the techniques of refs. $[57,58]$ to calculate the secondary decay. We identify some issues that can be accurately addressed and others that are less controlled and may contribute uncertainties that influence the final results. Later, we calculate the secondary decay of excited nuclei predicted by the statistical multi-fragmentation model and compare the final ground state yields to recent measurements.

\subsection{Levels and level densities}

To calculate the secondary decay corrections, one must specify both the high lying states that are mainly populated at freeze-out and the lower lying states whose populations increase as these excited nuclei decay towards the ground state nuclei that are experimentally measured. In the previous sections, the discussion was centered about the states that are populated at freeze-out. While in principle all nuclear states may be involved at freeze-out, the vast majority of fragments are excited to the particle unbound continuum.

The level densities in the unbound continuum influence the overall yield of unbound nuclei at freeze-out as well as the sequence and the number of particle decays. In principle, interactions between fragments and their surroundings modify the states and their excitation energies. The vanishing of the surface tension $\sigma(T)$ in the free energy expression at the critical temperature $T_{c}=18$ $\mathrm{MeV}$ reflects such considerations. Few experimental constraints on continuum level densities exist, however, even when the nuclei are isolated. Thus, uncertainties in the continuum level densities introduce uncertainties into the calculated results.

Following ref. [57], we represent the continuum level densities corresponding 
to the internal free energies in Eqs. 7 and 13 by the expression:

$$
\rho_{S M M}\left(E^{*}, J\right)=\rho_{S M M}\left(E^{*}\right) f(J, \sigma) .
$$

where $\rho_{S M M}\left(E^{*}\right)=\rho_{F G}\left(E^{*}\right) e^{-b_{S M M}\left(a_{S M M} E^{*}\right)^{3 / 2}}, b_{S M M}=0.07 A^{-1.82\left(1+\frac{A}{4500}\right)}$, $a_{S M M}=\frac{A}{\epsilon_{0}}+\frac{5}{2} \sigma_{0} \frac{A^{2 / 3}}{T_{c}^{2}}, J$ is the spin, $E^{*}$ is the excitation energy and $A$ is the mass of the fragment. For light and medium mass nuclei, $a_{S M M} \approx A / 8$. Here,

$$
\begin{aligned}
\rho_{F G}\left(E^{*}\right) & =\frac{a_{S M M}^{1 / 4}}{\sqrt{4 \pi}\left(E^{*}\right)^{3 / 4}} \exp \left(2 \sqrt{a_{S M M} E^{*}}\right), \\
f(J, \sigma) & =\frac{(2 J+1) \exp \left[-(J+1 / 2)^{2} / 2 \sigma^{2}\right]}{2 \sigma^{2}} \\
\sigma^{2} & \approx 0.0888 \sqrt{\left.A \cdot E^{*} / 8\right)} A^{2 / 3}
\end{aligned}
$$

and $E^{*}$ and $Z$ are the excitation energy and charge of the fragment. For further details, we refer the reader to ref. [57].

In contrast to the continuum level densities, the discrete level densities need no corrections for the influence of interactions because these levels become important only much later in the decay after the fragments have decoupled from their surroundings. For this purpose, we use the spectroscopic information of isolated nuclei with $Z<12$ where the information is available. For $12 \leq Z \leq 15$, low-lying states are not well identified experimentally and a continuum approximation to the discrete level density [59] was used. For all fragments with $Z \leq 15$ and excitation energies between the domains of discrete and continuum level densities, the level densities were smoothly interpolated $[57]$.

Where the experimental information for nuclei with $Z \leq 15$ is incomplete, values for the spin, isospin, and parity were chosen randomly in the decay calculations as follows: spins of 0-4 (1/2-9/2) were assumed with equal probability for even-A (odd-A) nuclei, parities were assumed to be odd or even with equal probability, and isospins were assumed to be the same as the isospin of the ground state. This simple assumption turns out to be sufficient since most of spectroscopic information is known for these low-lying states.

For excitation energies where little or no structure information exists, levels were assumed to be specified by the relevant level density expression. Groups of levels were binned together in discrete excitation energy intervals of $1 \mathrm{MeV}$ for $E^{*}<15 \mathrm{MeV}, 2 \mathrm{MeV}$ for $15<E^{*}<30 \mathrm{MeV}$, and $3 \mathrm{MeV}$ for $E^{*}>$ $30 \mathrm{MeV}$ to reduce the computer memory requirements. The results of the calculations do not appear to be sensitive to these binning widths. A cutoff energy of $E_{\text {cutoff }}^{*} / A=5 \mathrm{MeV}$ was introduced corresponding to a mean lifetime 
of the continuum states at the cutoff energy about $125 \mathrm{fm} / \mathrm{c}$. Where unknown, parities of these states were chosen to be positive and negative with equal probability and isospins were taken to be equal to the isospin of the ground state of the same nucleus. In this fashion, a table of states for nuclei with $Z \leq 15$ was constructed.

\subsection{Sequential decay algorithm}

Before sequential decay starts, hot fragments with $Z \leq 15$ were populated over the sampled levels in the prepared table according to the temperature. For the $i$ th level of a given nucleus $(\mathrm{A}, \mathrm{Z})$ with its energy $E_{i}^{*}$ and spin $J_{i}$, the initial population is,

$$
Y_{i}=Y_{0}(A, Z) \frac{\left(2 J_{i}+1\right) \exp \left(-E_{i}^{*} / T\right) \rho\left(E_{i}^{*}, J_{i}\right)}{\sum_{i}\left(2 J_{i}+1\right) \exp \left(-E_{i}^{*} / T\right) \rho\left(E_{i}^{*}, J_{i}\right)}
$$

where $Y_{0}$ is the primary yield summed over all states of nucleus $(\mathrm{A}, \mathrm{Z})$ and $\mathrm{T}$ is the temperature associated with the intrinsic excitation of the fragmenting system at breakup.

Finally all the fragments will decay sequentially through various excited states of lighter nuclei down to the ground states of the daughter decay products. The decay of fragments with $Z>15$ was calculated according to the fission model of ref. [60]. The subsequent decay of excited fission fragments with $Z \leq 15$ was calculated according to the Hauser-Feshbach algorithm described here. In this algorithm, eight decay branches of n, 2n, p, 2p, d, t, ${ }^{3} \mathrm{He}$ and alpha were considered for the particle unstable decays of nuclei with $\mathrm{Z} \leq 15$. The decays of particle stable excited states via gamma rays were also taken into account for the sequential decay process and for the calculation of the final ground state yields. If known, tabulated branching ratios were used to describe the decay of particle unstable states. Where such information was not available, the branching ratios were calculated from the Hauser-Feshbach formula [61],

$$
\frac{\Gamma_{c}}{\Gamma}=\frac{G_{c}}{\sum_{d} G_{d}}
$$

where

$$
\begin{aligned}
G_{d}= & \left\langle I_{d} I_{e} I_{d 3} I_{e 3} \mid I_{p} I_{p 3}\right\rangle^{2} \\
& \times \sum_{J=\left|J_{d}-J_{e}\right|}^{\left|J_{d}+J_{e}\right|} \sum_{l=\left|J_{p}-J\right|}^{\left|J_{p}+J\right|} \frac{1+\pi_{p} \pi_{d} \pi_{e}(-1)^{l}}{2} T_{l}(E)
\end{aligned}
$$


for a given decay channel $d$ (or a given state of the daughter fragment). $J_{p}$, $J_{d}$, and $J_{e}$ are the spins of the parent, daughter and emitted nuclei; $J$ and $l$ are the spin and orbital angular momentum of the decay channel; $T_{l}(E)$ is the transmission coefficient for the $l$ th partial wave. The factor $\left[1+\pi_{p} \pi_{d} \pi_{e}(-1)^{l}\right] / 2$ enforces parity conservation and depends on the parities $\pi= \pm 1$ of the parent, daughter and emitted nuclei. The Clebsch-Gordon coefficient involving $I_{p}, I_{d}$, and $I_{e}$, the isospins of the parent, daughter and emitted nuclei, likewise allows one to take isospin conservation into account.

For decays from empirical discrete states and $l \leq 20$, the transmission coefficients were interpolated from a set of calculated optical model transmission coefficients; otherwise a parameterization described in Ref. [59] was applied.

\section{Comparisons to data}

Even though the structure of the low-lying states of the fragments plays little role in properties of the hot system, these structure effects become critical when the fragments cool later by secondary decay. In the sequential decay algorithm described in the last section, in addition to more sophisticated level densities, empirical binding energies of the known nuclei are incorporated. Where the empirical masses are lacking, an improved mass formula [16,57] is employed. To be self-consistent, the same masses and level densities are used both in the thermodynamic model which produces the excited primary fragments and in the subsequent sequential decay. This self-consistency requirement appears to be necessary [16] for some observables. The resulting code which combines the thermodynamic model with the sequential decay algorithm is referred to as ISMM for improved Statistical Multifragmentation Model in the following sections of the report.

To illustrate the capabilities of the thermodynamic model, we calculate final ground state elemental and isotopic yields for systems with $A_{0}=168$ and $Z_{0}=75$ and $\mathrm{A}_{0}=186$ and $\mathrm{Z}_{0}=75$ at $T=4.7 \mathrm{MeV}$, corresponding to $E^{*} / A \approx 5$ $\mathrm{MeV}$. In all the following calculations, the freeze-out density is taken to be $1 / 6$ of the saturation density. These two systems were chosen because they have the same proton fractions as the combined systems formed in central ${ }^{112} \mathrm{Sn}+{ }^{112} \mathrm{Sn}$ and ${ }^{124} \mathrm{Sn}+{ }^{124} \mathrm{Sn}$ collisions, respectively. However, the overall size and excitation energy of these systems have been reduced below that of the corresponding compound nuclei to reflect the loss of particles and excitation energy to pre-equilibrium emission prior to the multi-fragment breakup. These parameters have not been adjusted to obtain a best fit of the data.

In the following, we illustrate the capability of this thermodynamic model to describe experimental charge, mass and isotopic yield distributions. We 
also compare experimental and calculated observables, such as the isotopic temperature and the isoscaling parameters, which are constructed from these yields.

\subsection{Charge and Mass Distributions}

Calculations of the mass distribution for excited primary fragments are shown in Fig. 15 for a system with $A_{0}=168$ and $Z_{0}=75$ at $T=4.7 \mathrm{MeV}$. The distributions of the primary fragments directly obtained from the thermodynamic model are shown as dashed lines with open points while the solid line with solid points represent the distributions of the final fragments after sequential decays. Certain differences between primary and final spectra can be expected. Heavier fragments formed in the multifragment stages decay to smaller fragments, shifting the distribution to lower masses. In addition, the decay produces a large increase in the hydrogen and helium particles, because these are the main products of the decay of the heavy fragments.

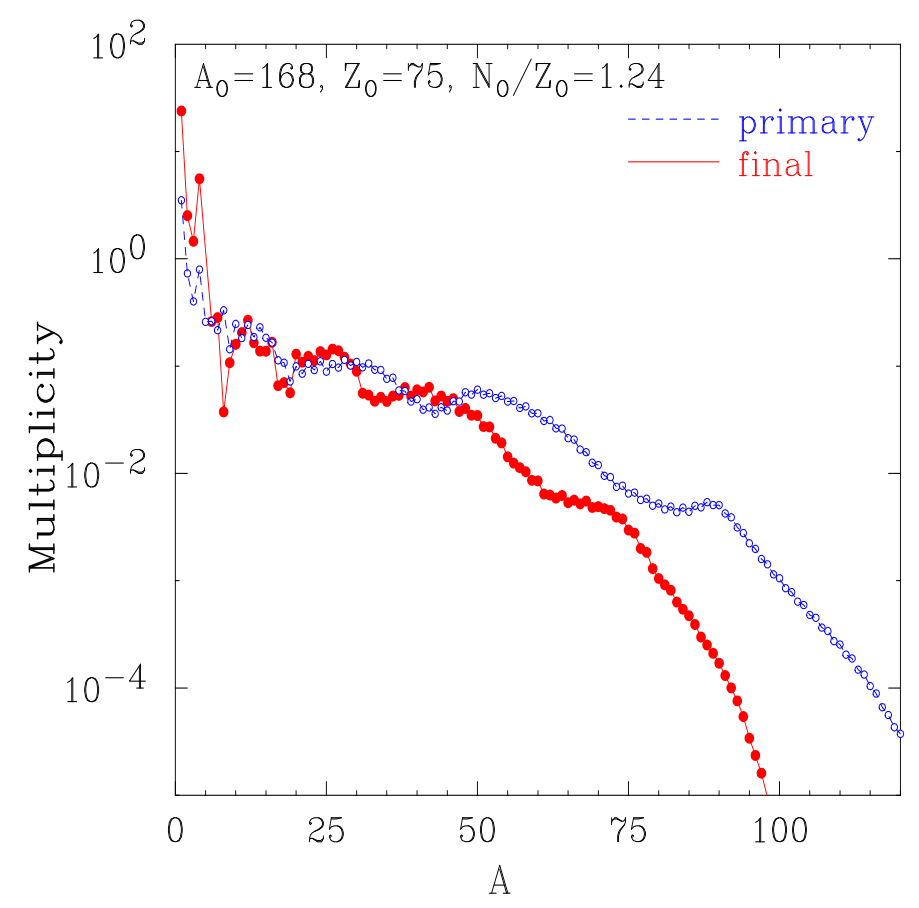

Fig. 15. Predicted mass distributions from the multifragmentation of a source nucleus with the mass number 168 and charge number 75. The open circles are primary yields and the closed circles are yields after secondary decay.

The differential multiplicities $d M / d \Omega$ for various masses with $A \leq 20$ are plotted in an expanded scale in Fig. 16 for both the $A_{0}=168$ and $A_{0}=186$ systems. For comparisons, experimental data obtained by averaging over $70^{\circ} \leq$ 
$\theta_{c m} \leq 110^{\circ}$ for central ${ }^{112} \mathrm{Sn}+{ }^{112} \mathrm{Sn}$ and ${ }^{124} \mathrm{Sn}+{ }^{124} \mathrm{Sn}$ collisions at $\mathrm{E} / \mathrm{A}=50 \mathrm{MeV}$ [62] are plotted as open and solid points in the left and right panels, respectively. The calculations reproduce many features of the mass distribution.

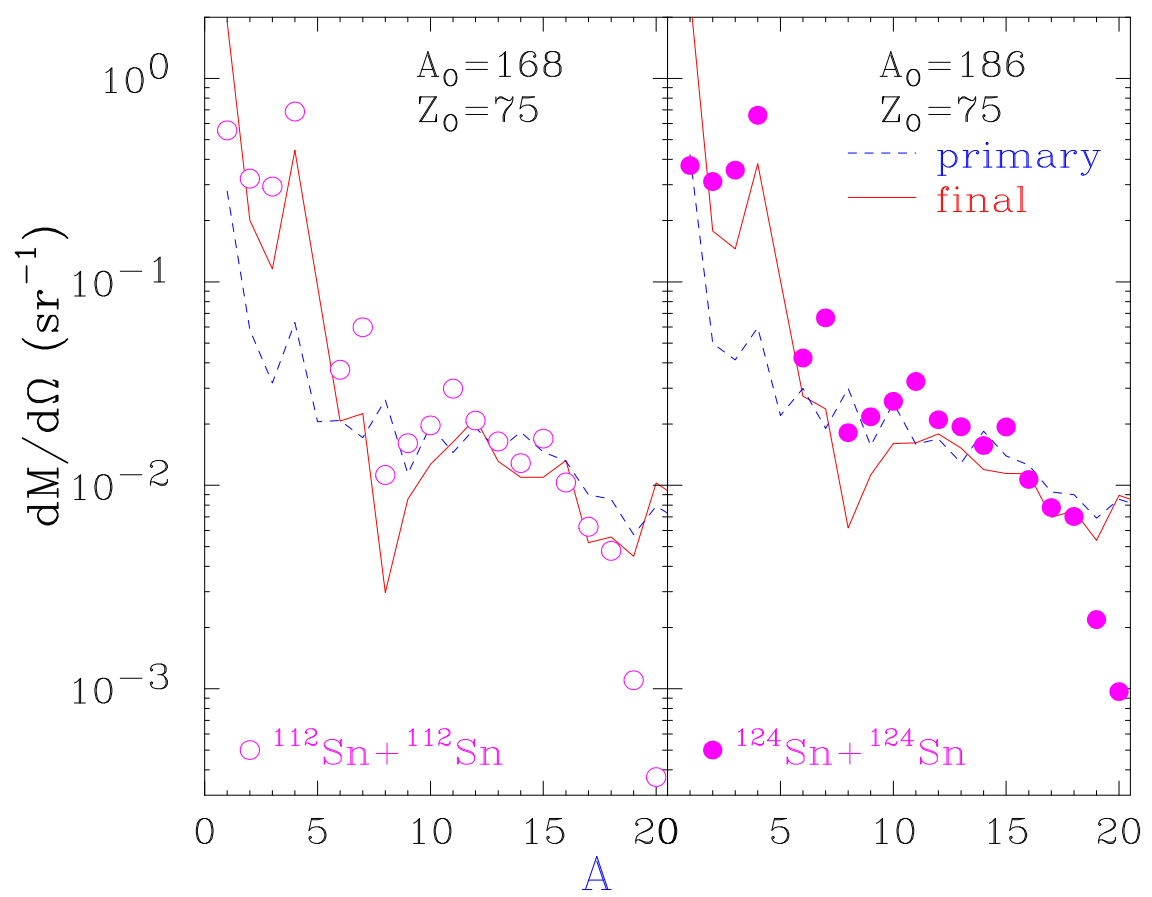

Fig. 16. Predicted mass distributions $(A \leq 20)$ from the multifragmentation of a source nucleus with $A_{0}=168$ and $Z_{0}=75$ (left panel) and $A_{0}=186$ and $Z_{0}=75$. The dashed lines are the predicted primary yields and the solid lines are predicted yields after secondary decay. For comparison, data from multifragmentation of central collisions of ${ }^{112} \mathrm{Sn}+{ }^{112} \mathrm{Sn}$ are shown as open symbols (left panel) and closed circles for ${ }^{124} \mathrm{Sn}+{ }^{124} \mathrm{Sn}$ reaction (right panel) [62].

The relative normalization of the calculation can be increased by increasing the size of the source or by making its angular distribution sideways peaked. The slope of the mass distribution can be made more steep by increasing the source temperature. There are indications that the experimental angular distributions are not isotropic and that pre-equilibrium emission mechanisms may contribute to the yields of the lighter fragments. Accordingly, we do not fit the calculations to the experimental data in this article, but defer such detailed analyses until more experimental data that can constrain such effects become available.

The charge distributions exhibit similar behavior as the mass distributions. For completeness, we include the charge distributions for the $A_{0}=168$ and $Z_{0}=75$ and $A_{0}=186$ and $Z_{0}=75$ in Figs. 17 and 18 . The same conventions for the mass distribution figures (Fig 15 and 16) are used. 


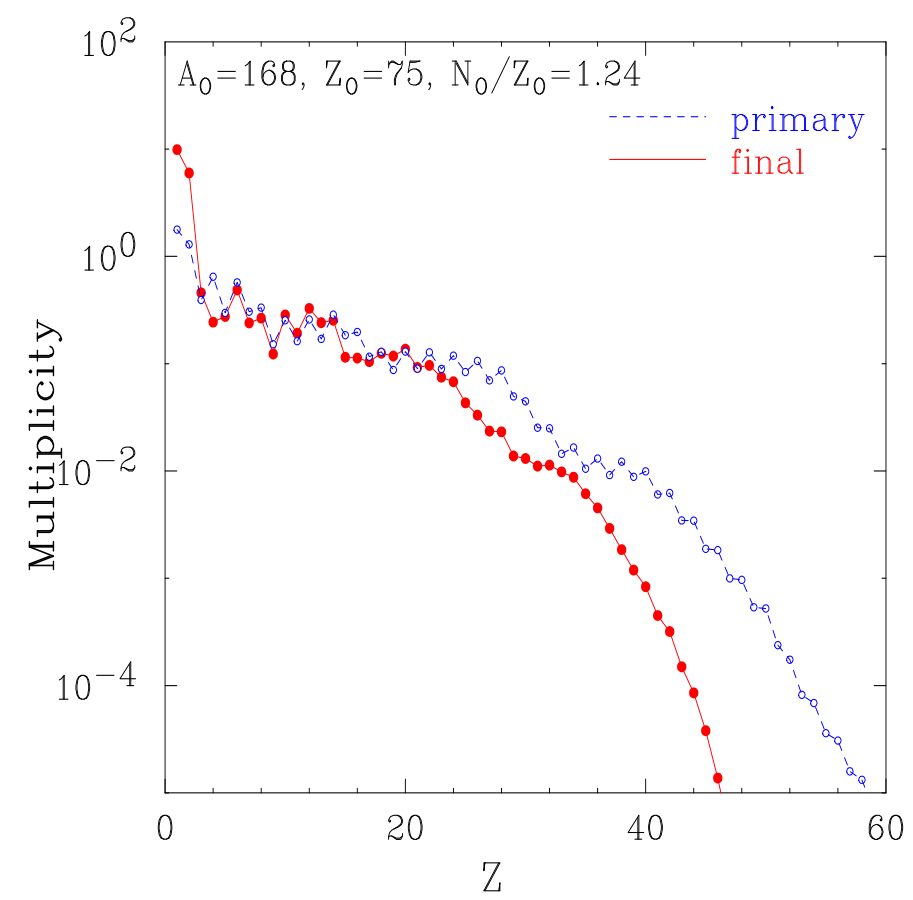

Fig. 17. Predicted charge distributions from the multifragmentation of a source nucleus with $A_{0}=168$ and $Z_{0}=75$. The open circles are primary fragment yields and the closed circles are after secondary decay.

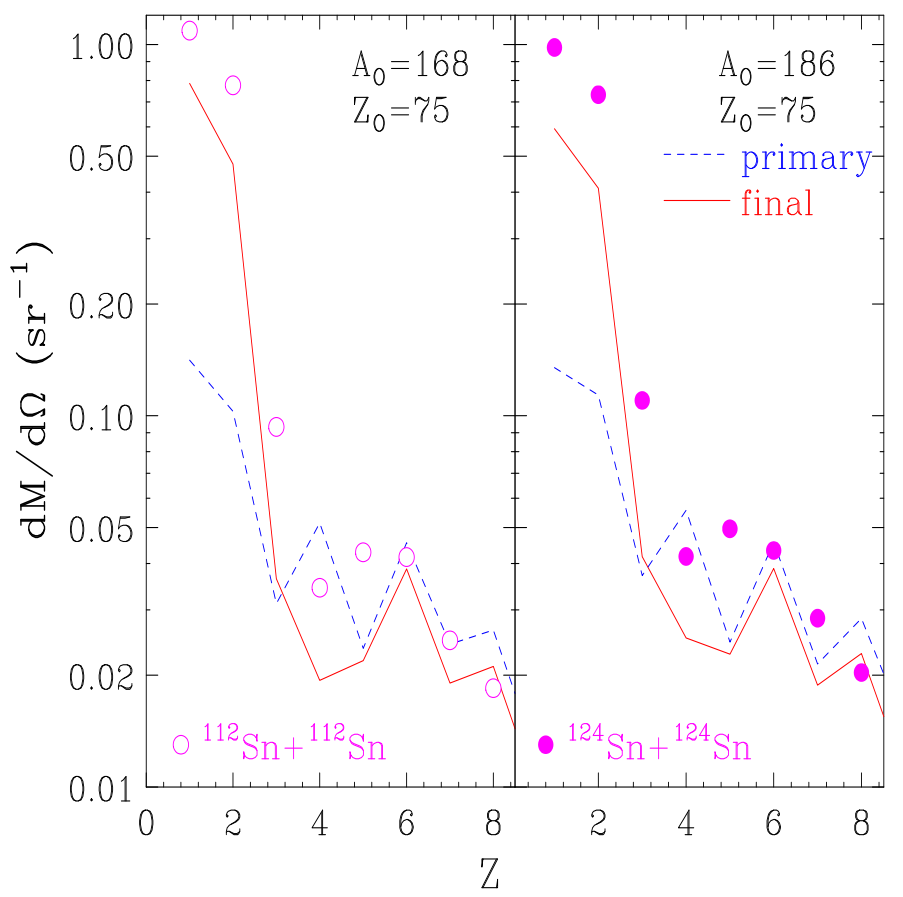

Fig. 18. Predicted charge distributions $(Z \leq 8)$ from the multifragmentation of source nuclei with $A_{0}=168$ and $Z_{0}=75$ (left panel) and $A_{0}=186$ and $Z_{0}=75$. The open and solid points are data from Ref.[62]. See Fig. 16 for explanations 
of symbols used.

In the break up calculations, the odd-even effects are evident. These occur because pairing and shell effects are not completely washed out in our level density expressions at a temperature of $T=4.7 \mathrm{MeV}$. As the secondary decay washes out such structures, these odd-even effects in the primary distribution have little or no effect on the final fragment distribution.

\subsection{Isotopic distributions}

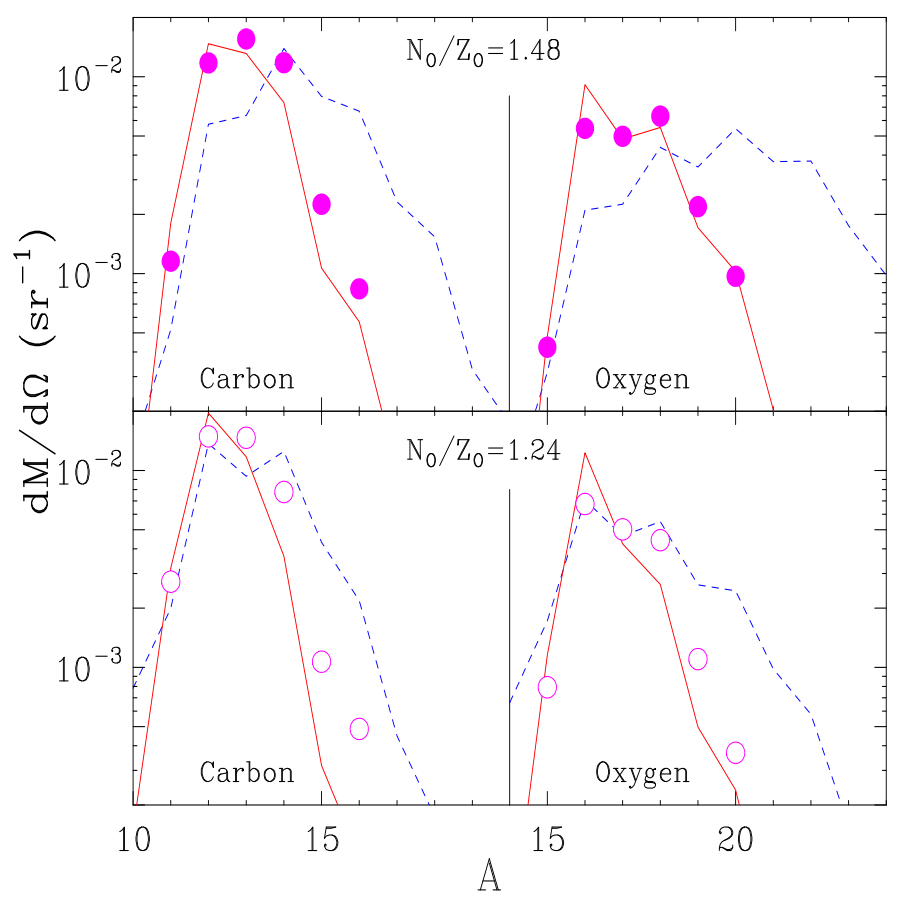

Fig. 19. Isotope distributions for Carbon and Oxygen fragments. The dashed and solid lines correspond to the predicted primary and final yields respectively. The open and solid points are from Ref. [62].

In Fig. 19, the isotopic distributions for carbon and oxygen isotopes are plotted for the two sources. Using the same convention as before, the dashed lines correspond to the distributions of the primary fragments while the solid lines represent the final distributions after sequential decay. As expected, the more neutron-rich system with $N_{0} / Z_{0}=1.48$ preferentially produces more neutronrich isotopes than the neutron deficient system with $N_{0} / Z_{0}=1.24$. In all cases, the primary distributions are much wider and more neutron-rich than the final distributions. The experimental isotope distributions (data points) agree more with the final results obtained after secondary decay than with the primary 
distributions. Nonetheless, the widths of the experimental distributions exceed those of the final distributions and are more neutron-rich. This suggests that the predicted corrections for secondary decay may be somewhat too large.

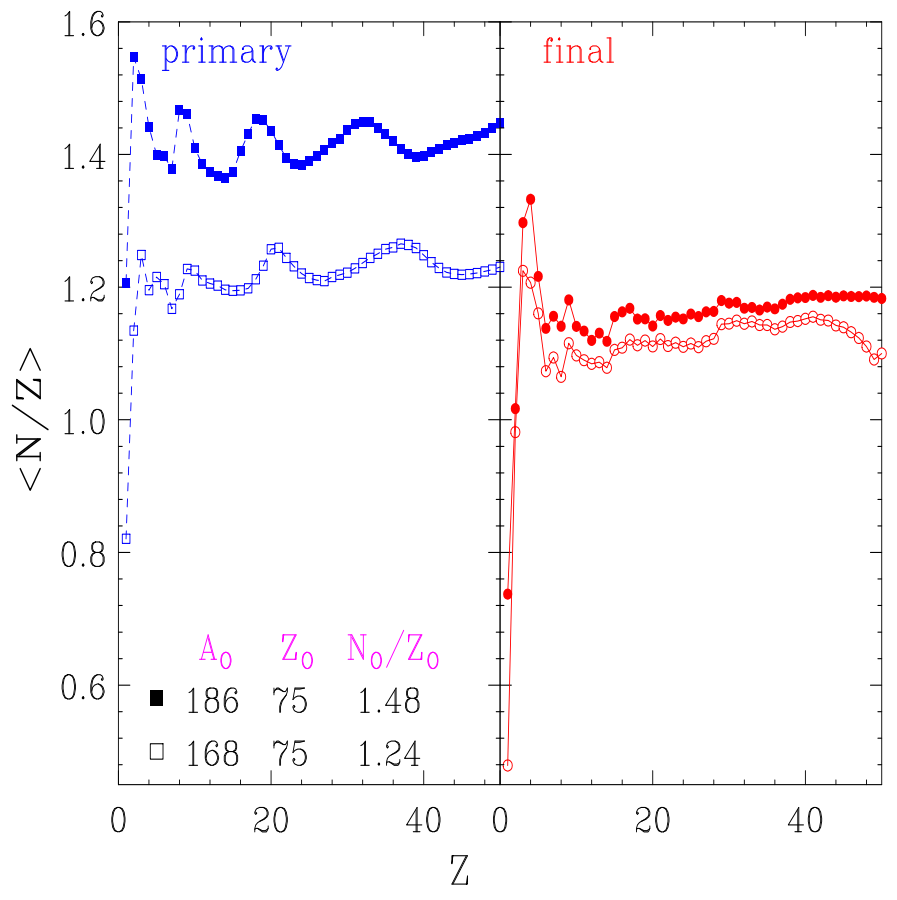

Fig. 20. The mean neutron to proton ratios as a function of the charge of the emitted fragment $Z$ for the two systems. The left and right panels correspond to the calculated results from the primary and final fragments.

The mean neutron to proton ratios $\langle N / Z\rangle$ for each element provides another observable with sensitivity to the isospin asymmetry dynamics of the reaction. The dependence of the calculated primary values on the $\langle N / Z\rangle$ of the total system is much stronger than that of the final values. This can be seen in Fig. 20 where the primary (left panel) and final (right panel) $<N / Z>$ values are compared for the two systems. The differences of the primary values for $<N / Z>$ of the two systems are large, reflecting the large difference in the initial isospin asymmetry of the two systems. The largest values for $\langle N / Z\rangle$ occur for $Z \approx 8,20$, etc., values corresponding to nuclei where one can have either closed proton or neutron shells. Such nuclei can remain comparatively well bound even for large value of $N / Z$. Both of these enhancement and the difference between the $\langle N / Z\rangle$ values for the two systems are diminished in the final distributions, which are both narrower and located closer to the valley of beta stability.

Fig. 21 shows measured and calculated primary and final values for $\langle N / Z\rangle$ as functions of the element number $Z$. The left and right hand panels provide the $\langle N / Z>$ values for the neutron-deficient and neutron-rich systems, re- 
spectively. The calculated final distributions reproduce the measured values well. It is rather curious that the experimental $\langle N / Z\rangle$ values exhibit the odd and even effects as a function $Z$. Such staggering is much less obvious in the neutron rich system. For reference, the $\langle N / Z\rangle$ for the abundances of naturally occurred isotopes are plotted as stars in both panels of the figure.

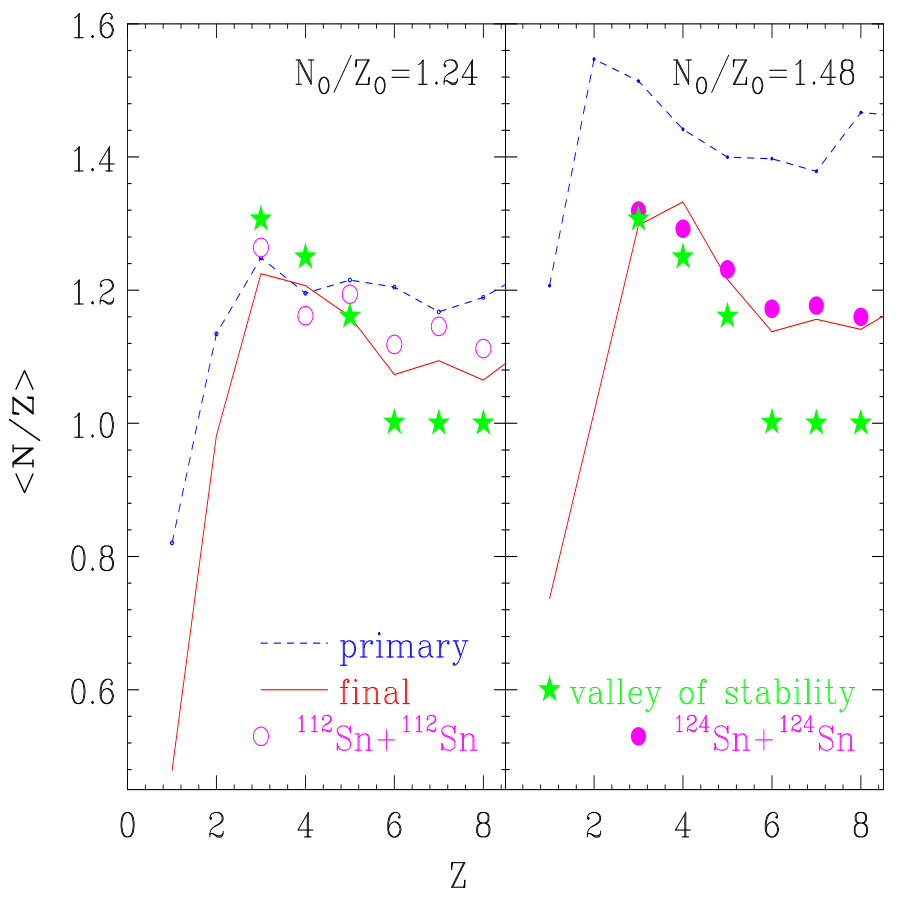

Fig. 21. The mean neutron to proton ratios as a function of the charge of the emitted fragment $Z$ for the neutron deficient (left panel) and neutron rich (right panel) systems. For comparison, data from the multifragmentation of central collisions of ${ }^{112} \mathrm{Sn}+{ }^{112} \mathrm{Sn}$ are shown as open symbols (left panel) and as closed circles for ${ }^{124} \mathrm{Sn}+{ }^{124} \mathrm{Sn}$ reaction (right panel)[62]. For reference, the mean $N / Z$ ratios from naturally occurring isotopes are shown as stars.

\subsection{Isoscaling}

The dependence of the isotopic distributions on the $N_{0} / Z_{0}$ of the colliding system can be more sensitively explored by the use of isotopic ratios $[62,63,64,65]$. In particular, the ratio, $R_{21}(N, Z)=Y_{2}(N, Z) / Y_{1}(N, Z)$, of yields from two different reactions, labelled here as 1 and 2, has been shown to exhibit an exponential relationship as a function of the isotope neutron number $N$, and proton number, $Z[62,63,64,65,66,67,68,69,70,71,72,73,74]$.

$$
R_{21}(N, Z)=C \cdot \exp (\alpha N+\beta Z)
$$


where $C$ is a normalization factor and $\alpha$ and $\beta$ are the isoscaling parameters.

Calculations with a variety of different statistical models show that the isoscaling relationship is strictly obeyed by the primary fragments in these models $[64,66,71]$. Surprisingly the isoscaling relationship is also obeyed by fragments produced in dynamical models such as the asymmetrized molecular dynamical model [70]. In all cases, the isoscaling parameters are related to the isospin asymmetry of the collisions and to the form of symmetry energy or, equivalently, asymmetry term of the EOS chosen in the model $[64,66,70,71,75]$.

Neglecting for simplicity the Coulomb interactions between fragments and environment, the exponential dependence of the isoscaling relationship can be easily understood from the expression for the yields for a fragment with neutron and proton numbers $N$ and $Z$ within the grand canonical limit of the present equilibrium model [76]:

$$
Y_{i}(N, Z)=V_{i} \frac{A^{3 / 2} q_{N, Z}(T)}{\lambda_{T_{i}}^{3}} \exp \left[\left(Z \mu_{p, i}+N \mu_{n, i}+B_{N, Z}\right) / T\right] .
$$

Here, $q_{N, Z}\left(T_{i}\right)$ represents the internal partition function of the fragment, $V_{i}$ the free volume of the system, $\lambda_{T}=\sqrt{2 \pi \hbar^{2} / m T_{i}}, m$ the nucleon mass and $\mu_{p, i}$ $\left(\mu_{n, i}\right)$ the chemical potential associated with free protons (neutrons) for the $i^{t h}$ reaction which produces a system at temperature $T_{i}$. If the temperature in the two reactions are expected to be the same (as in the Sn reactions described here), the chemical potentials $\mu_{p, i}$ and $\mu_{n, i}$ contain the only reaction dependent factors in this exponential. In this limit, $\alpha=\left[\mu_{n, 2}-\mu_{n, 1}\right] / T$ and $\beta=\left[\mu_{p, 2}-\mu_{p, 1}\right] / T$.

The symbols in Fig. 22 represent the isotopic ratios calculated by the canonical thermodynamic model described in this review. In Figs. 22 and 23, the following convention is adopted. We choose closed symbols and solid lines for even $\mathrm{Z}$ and open symbols and dashed lines for odd $\mathrm{Z}$ starting with $\mathrm{Z}=1$ for leftmost line. The lines are best fits of the calculated $R_{21}$ ratios to Eq. (33); the lines are essentially linear and parallel on this semi-log plot consistent with a single constant isoscaling parameter $\alpha_{\text {primary }}=0.50$. The spacing between these lines corresponds to the increase in $R_{21}$ for unit increases in $Z$; the observed equal spacing is consistent with a single constant isoscaling parameter $\beta_{\text {primary }}=-0.64$.

For comparison to the data, we only examine the isotope ratios where there are data with sufficient statistics. The symbols in the bottom panel of Fig. 23 represent the predicted isotopic ratios after sequential decays. The lines are nearly parallel to the lines in Fig. 22 on average and the isoscaling parameters $\alpha_{\text {final }}=0.46$ and $\beta_{\text {final }}=-0.52$ are comparable to the primary values. In detail especially when the isotopes away from the valley of stable nuclei are 
considered, the trends are not as clearly consistent with the isoscaling law as are the trends of the primary distribution. The larger change in the $\beta$ values may arise from the approximation of the Coulomb interaction used in the model. In the top panel, the data are shown as symbols. The experimental isoscaling parameters are $\alpha_{\text {data }}=0.36$ and $\beta_{\text {data }}=-0.42$. The slopes from the calculations are flatter suggesting that the temperature of $4.7 \mathrm{MeV}$ used as the input parameter in the model may be too low. However, if the temperature is increased so that the isoscaling predictions agree with the data, the other observable such as the mass and charge distributions as well as the isotope distributions may no longer agree. As stressed earlier, the current work is not to use the optimized set of model parameters but rather to compare the trends of data with the the model calculations. More constraints and study are needed to optimize the agreement with data.

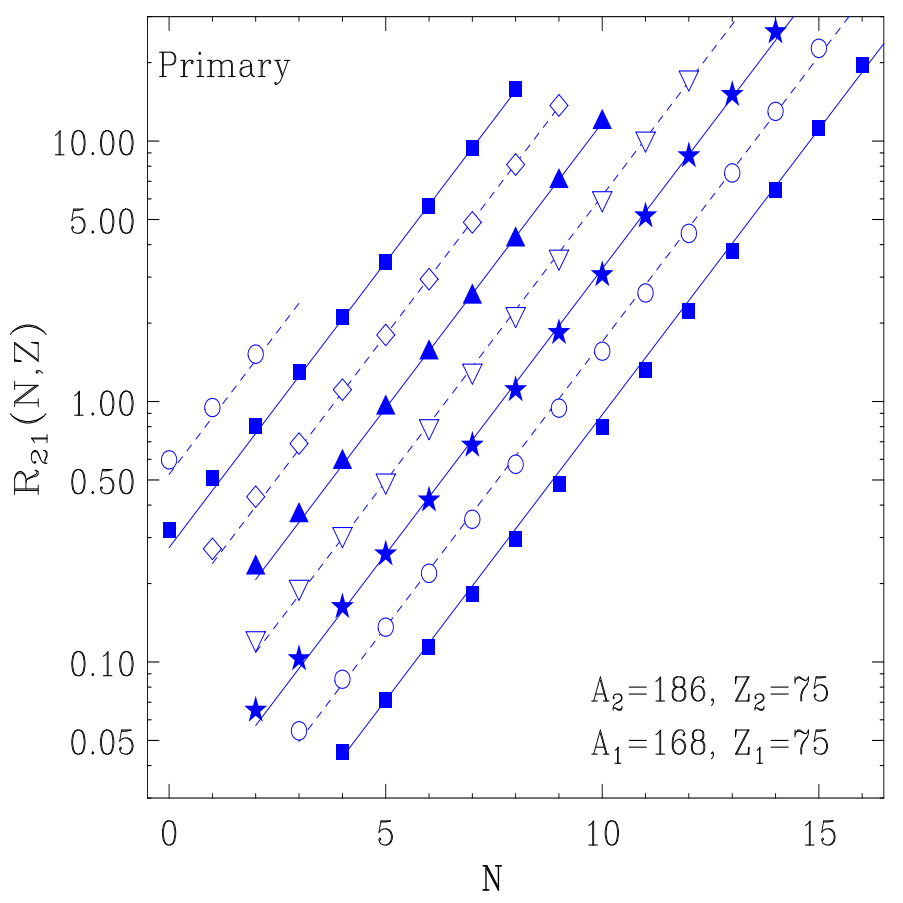

Fig. 22. Predicted yield ratios, $R_{21}(N, Z)=Y_{2}(N, Z) / Y_{1}(N, Z)$ from primary fragments for the two systems studied in this work. The lines are best fit to the symbols according to Eq. (33). Different lines correspond to $Z=1$ to 8 starting with the leftmost line with three points being $Z=1$. 


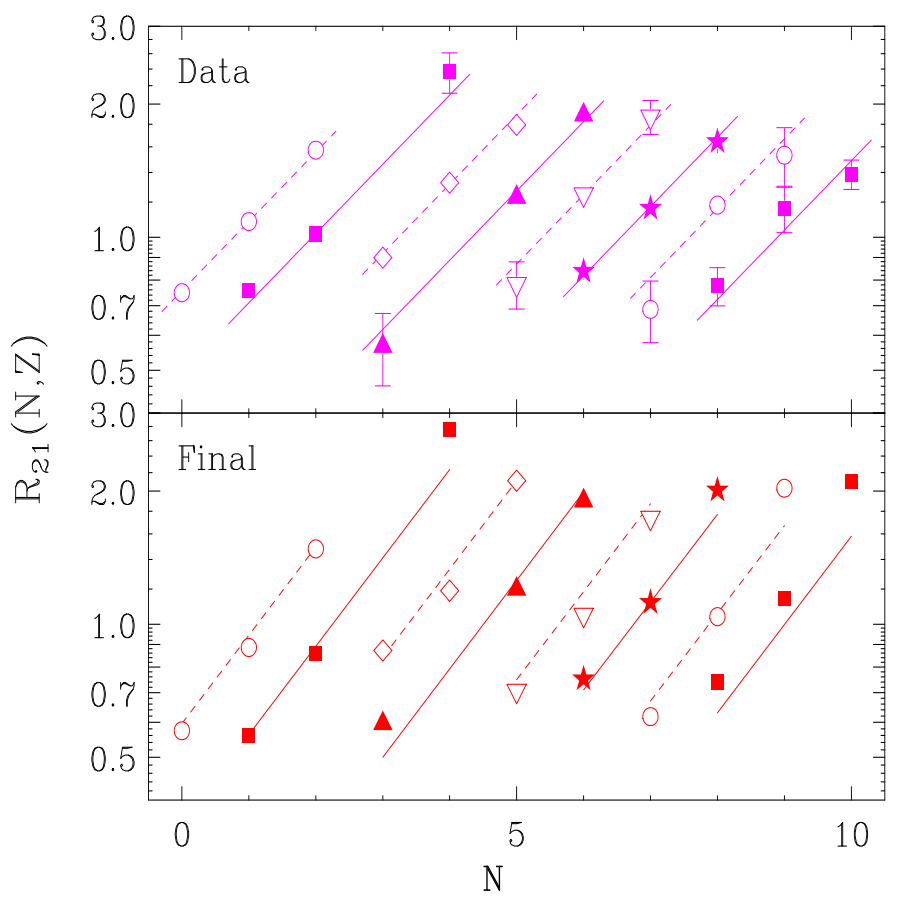

Fig. 23. Top Panel: Experimental isoscaling behavior exhibited by the central ${ }^{112} \mathrm{Sn}+{ }^{112} \mathrm{Sn}$ and ${ }^{124} \mathrm{Sn}+{ }^{124} \mathrm{Sn}$ collisions. The data are the nuclide yield ratios, $R_{21}(N, Z)$ from the two reactions plotted as a function of $\mathrm{N}$. The isotopes of different elements lie along different lines. The solid and dashed lines represent the best fit to Eq. (33). Bottom Panel: Predicted yield ratios, $R_{21}(N, Z)$ obtained from the final yields for the two systems studied in this work. The symbols and lines have the same convention as the data used in the top panel and Fig. 22.

\subsection{Isotopic temperatures}

Starting from the grand canonical expression for the yields (Eq. 34), it is also possible to construct a double ratio that minimizes the sensitivity to the isospin asymmetry while maximizing the sensitivity to the temperature. By doing so, one can construct an isotopic thermometer, whereby the temperature is extracted from a set of four isotopes produced in multifragment breakups as follows [77],

$$
T_{i s o}=\frac{\Delta B}{\ln (a R)}
$$

where 


$$
\begin{aligned}
R= & \frac{Y\left(A_{1}, Z_{1}\right) / Y\left(A_{1}+1, Z_{1}\right)}{Y\left(A_{2}, Z_{2}\right) / Y\left(A_{2}+1 . Z_{2}\right)}, \\
\Delta B= & B\left(A_{1}, Z_{1}\right)-B\left(A_{1}+1, Z_{1}\right) \\
& -B\left(A_{2}, Z_{2}\right)+B\left(A_{2}+1, Z_{2}\right),
\end{aligned}
$$

and

$$
a=\frac{\left(2 J_{Z_{2}, A_{2}}+1\right)\left(2 J_{Z_{1}, A_{1}+1}+1\right)}{\left(2 J_{Z_{1}, A_{1}}+1\right)\left(2 J_{Z_{2}, A_{2}+1}+1\right)}\left[\frac{A_{2}\left(A_{1}+1\right)}{A_{1}\left(A_{2}+1\right)}\right]^{3 / 2} .
$$

In this ratio derived from Eq. (36) for the ground state yields, $Y(A, Z)$ is the yield of a given fragment with mass $\mathrm{A}$ and charge $\mathrm{Z} ; B(A, Z)$ is the binding energy of this fragment; and $J_{Z, A}$ is the ground state spin of the nucleus. In the context of the grand canonical ensemble, Eq. (35) has been regarded as an effective or "apparent" temperature that may differ somewhat from the true freezeout temperature $T$ due to the influence of secondary decay and other cooling mechanisms.

The influence of secondary decay on the isotopic temperatures can be clearly observed because it leads to variations in the values for the temperature that depend on the isotopes used to construct the ratio. The variations are universal, observed in many different reaction systems and thus can be used to assess the effectiveness of sequential decay models. One origin of these variations is the feeding from higher lying particle bound states. Such effects can be modeled by changing the value for the statistical factor "a" and making it temperature dependent. This and additional feeding from the decay of heavier particle unbound nuclei can be modeled by the secondary decay formalism described in the previous section.

To illustrate the influence of secondary decay on isotope temperature measurements, measured and calculated final temperatures have been extracted from double ratios of $Z=2-8$ fragments and plotted in Fig. 24. To reduce the influence of secondary decay, we include only isotope thermometers with large values for $\Delta B$ in this figure. This requirement restricts comparisons to three types of thermometers: a.) $T_{i s o}\left({ }^{3,4} \mathrm{He}\right)$ with $Z_{2}=2, A_{2}=3$, b.) $T_{i s o}\left({ }^{11,12} C\right)$ with $Z_{2}=6, A_{2}=11$, and c.) $T_{i s o}\left({ }^{15,16} O\right)$ with $Z_{2}=8, A_{2}=15$. We note that the thermometer (a) involves the light particle pair ${ }^{3,4} \mathrm{He}$ while thermometers (b) and (c) concern only intermediate mass fragments with $Z=3-8$. The solid lines show corresponding ISMM predictions for these three types of thermometers as a function of $A_{1}$.

Similarities in the variations of the calculated and measured temperatures allow insight into their origin. Each panel of Fig. 24 corresponds to fixed values of $Z_{2}$ and $A_{2}$; the observed variations in $T_{i s o}$ are therefore correlated with $Z_{1}$ and $A_{1}$. The highest values for $T_{\text {iso }}$ involve ${ }^{10} \mathrm{Be}\left(Z_{1}=4, A_{1}=10\right)$ and ${ }^{18} \mathrm{O}\left(Z_{1}=8\right.$, 
$\left.A_{1}=18\right)$. The calculations attribute this increase to enhancements in the yields of these nuclei due to $\gamma$-ray feeding from their many low lying particle bound states [80]. Other thermometers in Fig. 24 provide temperature values that are significantly lower than those involving ${ }^{10} \mathrm{Be}$ and ${ }^{18} \mathrm{O}$. Most thermometers are significantly lower than the primary temperature of $4.7 \mathrm{MeV}$, depicted by the horizontal dashed line in the three panels.

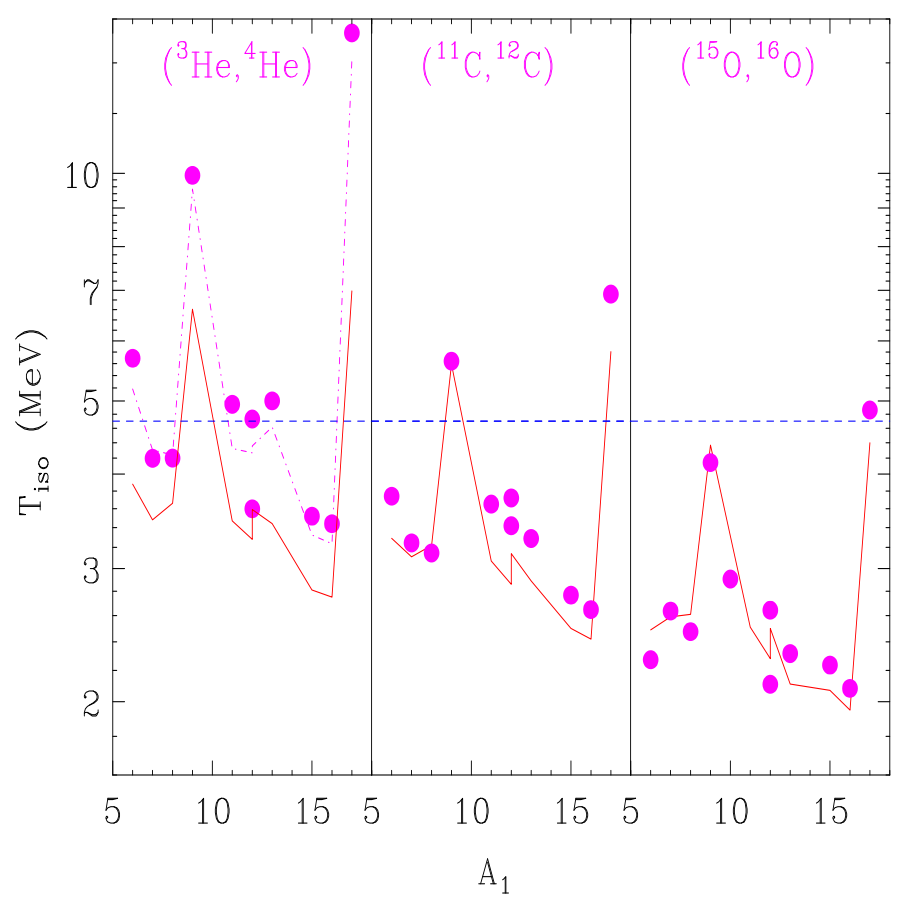

Fig. 24. Isotopic temperatures extracted from three types of thermometers. Experimental data are shown as symbols. The lines are calculations. For reference, the input primary temperature of $4.7 \mathrm{MeV}$ is shown as the horizontal dashed lines. (See text for details on the dot-dashed line in the left panel.)

Both calculated and measured values display a $\mathrm{Z}$ or $\mathrm{A}$ dependence in $T_{i s o}$. Calculated values for $T_{i s o}\left({ }^{15,16} O\right)$ are about $0.5 \mathrm{MeV}$ lower than those for $T_{i s o}\left({ }^{11,12} \mathrm{C}\right)$, which are about $0.2 \mathrm{MeV}$ lower than $T_{i s o}\left({ }^{3,4} \mathrm{He}\right)$. There is also a trend for isotopic temperature values to decrease as a function of $A_{1}$. The calculated decrease of $T_{i s o}$ with $A_{1}$ and $A_{2}$ reflect the increasing importance of multi-step secondary decay contributions to the yields of these heavier nuclei. Such multi-step decays make the system appear cooler because the final ground state nuclei originate from the decay of an ensemble of unstable nuclei that are less excited than the original ensemble.

We note that the experimental $T_{i s o}\left({ }^{3,4} \mathrm{He}\right)$ temperatures (solid symbols in the left panel) are systematically higher than the corresponding ISMM values (solid line). As these thermometers derive their sensitivity to the temperature from the large binding energy difference between ${ }^{3} \mathrm{He}$ and ${ }^{4} \mathrm{He}$, the difficulty in 
reproducing these quantities may arise if there are significant pre-equilibrium production mechanisms for light particles such as ${ }^{3} \mathrm{He}$ [80]. To illustrate this effect, we assumed that $2 / 3$ of the measured ${ }^{3} \mathrm{He}$ yield is of a non-thermal origin. This increases the ${ }^{3} \mathrm{He}$ yield by a factor of three; calculations including this pre-equilibrium enhancement are shown as the dot dashed line in the left panel. The success of this resolution of the discrepancies between $T_{i s o}\left({ }^{3,4} \mathrm{He}\right)$ and $T_{i s o}\left({ }^{11,12} C\right)$ suggests that it may be necessary to make careful estimations of the contributions from pre-equilibrium emission before isotope temperature measurements involving $T_{i s o}\left({ }^{3,4} \mathrm{He}\right)$ will be fully accurate.

\section{Summary}

The canonical version of the thermodynamic model has helped clarify many aspects of intermediate energy heavy ion collisions. The obvious advantage is that, as opposed to the grand canonical model, it has an exact number of particles. The predictions of the grand canonical model (which really applies to very large systems) can differ very significantly from those of the canonical model specially in the intermediate energy regime. The canonical model helps us to understand the order of phase transition, the caloric curve and the possibility of negative specific heat. The model gives quantitative fits to experimental data on isotopic yields and the phenomenon of isoscaling, now

so well established in intermediate energy heavy ion collisions. The virtue of the model is also its simplicity. Most of the calculations reported in this work can be carried out quite easily.

\section{Acknowledgement}

This work is supported by Natural Sciences and Enginnering Research Council of Canada, by Quebec Department of Education, National Science Foundation under Grant No. PHY-01-10253 and US Department of Energy Grant No. DEFG02-96ER40987.

\section{A Equilibrium, reactions and reaction rate time scales}

A basic assumption of statistical models is that equilibrium is reached in the time scale of the reaction. For fragment or composite particle distributions a complex set of reactions takes places $[81,82]$. The processes involved in the 
collision of heavy ions can be modeled in a manner that is similar to nucleosynthesis in a dense, heated and evolving system such as in the expansion of the early universe and in supernovae explosions. The starting point of such a description is then a dense and heated system of neutrons and protons which combine through a set of reactions to make the composite nuclei from the lightest nuclei such as deuterons, alpha particles,.. , all the way up to much heavier and complex nuclei. By way of illustration and also for contrast, the nucleosynthesis in the early universe occurs through a set of two body reactions with the first element of the chain being an electromagnetic radiative capture of a neutron plus proton to a deuteron with an emitted photon carrying away the excess energy. After this first electromagnetic process, light elements are produced by a sequential set of two body reactions such as $d+d \rightarrow H e^{3}+n, d+d \rightarrow t+p, t+d \rightarrow H e^{4}+n, \ldots$ Nuclei up to $L i$ are believed to be produced at their equilibrium concentration in big bang nucleosynthesis models. The abundance of heavy elements comes from processes involved in supernovae. The study of these processes is the area of nuclear astrophysics and heavy ion collisions offer the opportunity to study similar processes and phenomena in the laboratory.

In heavy ion collisions, electromagnetic processes are too slow over the time scale of the collision to produce the observed distribution of composites or produced particles. A typical time scale of the collision is $10^{-22} \mathrm{sec}$ or $30 \mathrm{fm} / \mathrm{c}$ which is much shorter than any electromagnetic process time scale. Densities in heavy ion collisions can be high enough for a three body process to occur such as $n+p+N \rightarrow d+N$, where the nucleon $N$ can carry away the excess energy. At very high energies, meson production processes occur, so that a $d$ is formed in radiative pion emission of $n+p$. Heavier composite particles evolve through reactions such as those listed above. However, it should be noted that because of possible very high initial densities, multi body processes can occur besides two body processes even for composites heavier than the deuteron. These only enhance the approach to equilibrium. At RHIC energies, particle production becomes very important, and reactions leading to new particles have been studied $[82,83]$.

As an example of a reaction rate approach consider the formation of a deuteron through the process $p+n+N \rightarrow d+N$. The time evolution of the deuteron density $\rho_{d}$ can be obtained from an equation involving the proton density $\rho_{p}$, neutron density $\rho_{n}$, and nucleon density $\rho_{N}$ :

$$
\frac{d \rho_{d}}{d t}=\left[\rho_{p} \rho_{n}\left[\left(\frac{\rho_{d}}{\rho_{n} \rho_{p}}\right)_{e q}-\rho_{d}\right] \rho_{N}\langle\sigma[N+d \rightarrow n+p+N] \times v\rangle\right.
$$

Here $\left(\frac{\rho_{d}}{\rho_{n} \rho_{p}}\right)_{e q}$ is the equilibrium ratio of the densities of $d$ 's to $n$ 's and $p$ 's and is a function of temperature. The \langle\rangle term in A.1 involves the product 
of the breakup cross section of deuterons induced by nucleons and $v$, which is the relative velocity of the $N$ and $d$ pair. This product is averaged over the velocity distribution of the pair. In obtaining the expression in A.1 we used detailed balance which relates the forward rate for the formation process $p+n+N \rightarrow d+N$ to the backward rate of the break up or absorption process $d+N \rightarrow p+n+N$. Equilibrium is reached when the forward rate is equal to the absorption rate. Initially, the deuteron density is being built up by forward processes which involves the product of proton, neutron and nucleon densities, but later in this time evolution deuterons will start to be absorbed by backward processes which involve the newly formed deuterons and the existing nucleons. Once equilibrium is reached these underlying processes vanish in the description of the deuteron density, which is now described by phase space factors with temperature and volume playing a dominant role. Large volumes reduce composites since nucleons are less likely to be near each other to combine and high temperatures increase break up probabilities. Binding energy terms appear as Boltzmann factors and enhance composite densities.

We can question whether rates are fast enough to produce equilibrium distributions. To answer this question we consider the following simplified expression for a reaction rate: $\rho_{N} \times \sigma \times v$. For $\rho_{N}$ we take nuclear matter density or 0.15 nucleons $/ \mathrm{fm}^{3}$. Typically temperatures are 10 's of $\mathrm{MeV}$ for medium energy collisions and a temperature of $10 \mathrm{MeV}$ has a kinetic energy of $15 \mathrm{MeV}=(1 / 2) m(v / c)^{2}$. For $v / c=1 / 5$, a cross section $\approx 1 \mathrm{fm}^{2}$ will have a rate $10^{22} / \mathrm{s}$. The reciprocal of this rate is the reaction rate time scale which is $10^{-22} \mathrm{~s}$. Thus, a cross section of $1 \mathrm{fm}^{2}$ will have a reaction rate time scale that is equal to the characteristic time scale of the collision. Under these circumstances equilibrium will be reached.

Next, consider the prototype two body reaction $A+B \rightarrow C+D$. The rate of growth of the density of $C$ can be related to the chemical activity $A=$ $\mu_{A}+\mu_{B}-\mu_{C}-\mu_{D}$, where $\mu_{A}$ is the chemical potential of $A$, etc. Specifically, the time evolution of the density of $C$ is

$$
\frac{d \rho_{C}}{d t}=\rho_{A} \rho_{B}\langle\sigma[A+B \rightarrow C+D] \times v\rangle(1-\exp [-A / T])
$$

At equilibrium $\mu_{A}+\mu_{B}=\mu_{C}+\mu_{D}$. Thus, the factor $(1-\exp [-A / T]) \rightarrow 0$. Near equilibrium $A<<T$ and $(1-\exp [-A / T]) \rightarrow A / T$. In this limit the reaction rate eq.(A.2) is linear in the chemical activity $A$. Such linear connections are known as Onsager relations where the chemical activity acts as a generalized force, $X$, and the left hand side of eq.(A.2) is interpreted as a generalized velocity $J$. Then $J=L X$, where $L$ is the proportionality constant between $J$ and $X$. Far from equilibrium, this linear relation is no longer valid since $A$ is, in general, not small compared to $T$. 
As a final consideration in discussing reaction rates we note that if the equilibrium concentration of the particle of interest is small, then the reaction rate constant is somewhat more complicated than the simplified expression used above. To illustrate this situation we mention the case of pion production. For example, for the reaction $N+N \rightarrow N+N+\pi$, the rate equation for the pion density is:

$$
d\left(\rho_{\pi}\right) / d t=\left[\rho_{N}^{2}-\left(\rho_{N}^{2} * \rho_{\pi} /\left(\rho_{\pi}\right)_{e q}\right] \times\langle\sigma v\rangle .\right.
$$

Here, $\left(\rho_{\pi}\right)_{e q}$ is the equilibrium pion density which depends on temperature. This rate equation can be solved to give $\rho_{\pi}(t)=\left(\rho_{\pi}\right)_{e q} *(1-\exp [-\lambda \times t])$. The rate constant is

$$
\lambda=\langle\sigma v\rangle \times \rho_{N}^{2} /\left(\rho_{\pi}\right)_{e q}
$$

The result of eq.(A.4) differs from the simplified reaction rate used above by an important factor $\rho_{N} /\left(\rho_{\pi}\right)_{e q}$. This factor can be very large when the equilibrium density of pions is small compared to the nucleon density. It was one of the reasons why the results of [82] led to the conclusions that pions would be in chemical equilibrium, a result which differed from a previous result in [84]. While low equilibrium concentration can enhance reaction rate constants and reduce equilibration time scales, some examples of other enhancement factors are the presence of two or more channels to the final state, the presence of secondary processes, high densities which allow multiparticle production processes above the two body type just considered. For example, the time scale for kaon production is considerably reduced through pion induced reactions, where the pions are copiously produced in the initial nucleon-nucleon collisions as first noted in [82].

\section{B Antisymmetry and all that}

Our whole discussion started from eq.(2) in section 2 which then led to eq.(5), the recursive formula. Eq.(2) is not quantum mechanical. The partition function of $n_{i}$ particles takes this simple form only under situation of low density and high temperature. We argue here that the approximation is quite good for intermediate energy heavy ion collisions.

We start with qualitative arguments. The volumes used are about three times or more of the normal volume. At low temperature $(\approx 4 \mathrm{MeV})$ where one might imagine the approximation to fail, it survives because many composites appear 
thus there is not enough of any particular species to make (anti)symmetrisation an important issue. At much higher temperature the number of protons and neutrons increase but as is well-known the $n$ ! correction takes the approximate partition function towards the proper one at high temperature. In a hypothetical world, the problem could get very difficult. Such a scenario would arise if the physics was such that at low temperature we only had neutrons and protons and no composites. An even worse situation would be if we had only neutrons (or protons). With these preliminaries let us proceed to estimate quantitatively the errors involved in actual cases that one might encounter in intermediate energy heavy ion collisions.

The recursive relation eq. (5) is not limited to the approximation of eq.(2). It is shown in [18] that by regarding the grand partition function (in our case this grand partition function incorporates correct (anti)symmetry among particles) as the generating function of the canonical partition function one derives a relation like eq.(5)

$$
Q_{N}(\beta)=\frac{1}{N} \sum_{k=1}^{N} k x_{k} Q_{N-k}(\beta)
$$

where $x_{k}$ is not a one-particle partition function but is to be obtained from an expansion of the grand partition function. We illustrate this with first the example of only protons filling up orbitals $i, j, k, \ldots$ in a box. Now

$$
\begin{aligned}
\ln Q_{g r}(\beta, \mu) & =\sum_{i} \ln \left(1+e^{\beta \mu-\beta \epsilon_{i}}\right) \\
& =\sum_{i} \sum_{j} \frac{(-)^{j-1}}{j} e^{j\left(\beta \mu-\beta \epsilon_{i}\right)}
\end{aligned}
$$

The coefficient of $e^{\beta \mu k}$ is $x_{k}$ which then gives $x_{k}=\frac{(-)^{k-1}}{k} \sum_{i} e^{-k \beta \epsilon_{i}}$ When this expression for $x_{k}$ is used in eq. B.1 it generates the correct partition function. Orbitals are given occupancies greater than one and then eliminated by subtraction. This can lead to severe round-off errors when applied to degenerate Fermi systems but will not affect the application we envisage here. The number of of protons is given by

$$
Z=\left(\frac{x_{1} Q_{Z-1}}{Q_{Z}}+\frac{2 x_{2} Q_{Z-2}}{Q_{Z}}+\ldots \frac{Z x_{Z} Q_{0}}{Q_{Z}}\right) / Z
$$

The value of $Q_{0}$ is 1 .

Anticipating generalisation we will call $x_{k}$ in the above case $y_{1,0}^{[k]}$. The subscript 1, 0 means it is a "composite" with one proton and no neutron. The superscript 
$k$ means it is obtained from the $k$-th term in the expansion; $y_{1,0}^{[k]}$ will contribute to $x_{k, 0}$.

If instead we had a boson, deuterons for example, we would have

$$
\begin{aligned}
\ln \left[Q_{\text {gr.can }}\left(\beta, \mu_{p}, \mu_{n}\right)\right] & =\sum_{i}-\ln \left(1-e^{\beta \mu_{p}+\beta \mu_{n}} e^{-\beta \epsilon_{i}}\right) \\
& =\sum_{i} \sum_{j} \frac{1}{j} e^{j\left(\beta \mu_{p}+\beta \mu_{n}-\beta \epsilon_{j}\right)}
\end{aligned}
$$

Thus in the case of deuterons $y_{1,1}^{[k]}$ (which would contribute to $x_{k, k}$ ) is given by $\sum_{i} \frac{1}{k} e^{-k \beta \epsilon_{i}}$.

We can treat an assembly of protons, neutrons, deuterons, tritons...etc. The recursive relation if the dissociating system has $Z$ protons and $N$ neutrons is

$$
Q_{Z, N}=\frac{1}{Z} \sum_{i=1, Z, j=0, N} i x_{i, j} Q_{Z-i, N-j}
$$

The average number of a composite with $i_{1}$ protons and $i_{2}$ neutrons is given by

$$
<n_{i_{1}, i_{2}}>=y_{i_{1}, i_{2}}^{[1]} Q_{Z-i_{1}, N-i_{2}} / Q_{Z, N}+2 y_{i_{1}, i_{2}}^{[2]} Q_{Z-2 i_{1}, N-2 i_{2}} / Q_{Z, N}+\ldots
$$

Unless one is in an extreme degenerate fermi system, one can evaluate the $y$ factors by replacing sums with integration. For example, $y_{1,0}^{[n]}=\frac{(-)^{n-1}}{n} \sum_{i} e^{-n \beta \epsilon_{i}}$ where the sum is replaced by $\int e^{-n \beta \epsilon} g(\epsilon) d \epsilon=2 \frac{V}{h^{3}}\left(\frac{2 \pi m}{n \beta}\right)^{3 / 2}$. Here $V$ is the available volume. We have included the proton spin degeneracy; $m$ is the proton mass. For the deuteron, $y_{1,1}^{[k]}=\frac{1}{k} \int e^{-k \beta \epsilon} g(\epsilon) d \epsilon$. This is $3 \times 2^{3 / 2} \frac{V}{h^{3}}\left(\frac{2 \pi m}{\beta}\right)^{3 / 2} \frac{e^{k \beta E_{b}}}{k^{5 / 2}}$ where $E_{b}$ is the binding energy of the deuteron. It is clear how to compute contributions from other composites.

We test the accuracy of the yields as calculated throughout the main text by comparing with a calculation where the complete theory of symmetrisation and antisymmetrisation is used. Subject only to the approximation that summation over discrete states has been replaced by an integration over a density of states, the calculation is exact. The results are taken from [18]. We take the dissociating system to have $Z=25$ and $N=25$. The lowest temperature considered is $3 \mathrm{MeV}$ (one might argue that at lower temperature a model of sequential decay is more appropriate). The highest temperature shown is 30 $\mathrm{MeV}$. We take a freeze-out volume in which the composites can move freely as three times the volume of a normal nucleus with 50 nucleons. Aside from neutrons and protons we allow the possibility of composites. Excited states of the 
Table B.1

Comparision of claculations of average yields and E/A. By exact we mean a calculation with proper symmetry. Sum over discrete orbitals in a box has been replaced by integration as is the usual practice.

\begin{tabular}{cccccccccc}
\hline Calc & $p$ & $n$ & $d$ & $t$ & ${ }^{3} H e$ & ${ }^{4} H e$ & $Z>12$ & $\begin{array}{c}\text { Temp. } \\
(\mathrm{MeV})\end{array}$ & $\begin{array}{c}E / A \\
(\mathrm{MeV})\end{array}$ \\
\hline approx & 0.307 & 0.032 & 0.050 & 0.007 & 0.054 & 0.679 & 0.945 & 3 & -7.863 \\
exact & 0.306 & 0.031 & 0.051 & 0.007 & 0.053 & 0.696 & 0.945 & 3 & -7.861 \\
approx & 1.174 & 0.898 & 1.177 & 0.560 & 0.641 & 2.489 & 0.051 & 6 & -4.117 \\
exact & 1.117 & 0.856 & 1.195 & 0.553 & 0.638 & 2.573 & 0.050 & 6 & -4.135 \\
approx & 4.127 & 3.955 & 4.812 & 2.099 & 2.052 & 1.985 & 0.000 & 12 & 4.401 \\
exact & 3.860 & 3.696 & 4.941 & 2.090 & 2.051 & 2.021 & 0.000 & 12 & 4.308 \\
approx & 10.937 & 10.893 & 7.664 & 1.686 & 1.650 & 0.379 & 0.000 & 30 & 28.914 \\
exact & 10.512 & 10.468 & 7.885 & 1.732 & 1.696 & 0.395 & 0.000 & 30 & 28.844 \\
\hline
\end{tabular}

composites were not allowed (they could have been included but the purpose of the exercise was to compare two models: calculations without the inclusion of excited states were sufficient to reach conclusions). Spins and binding energies for deuteron, triton, ${ }^{3} \mathrm{He}$ and ${ }^{4} \mathrm{He}$ are taken from experiments. For higher mass composites the binding energy is taken from empirical mass formulas. For fermions, spin 1/2 was assumed and for bosons spin 0 was assumed. For each $Z$ we take $N=Z-1, Z$, and $Z+1$. We present in the table average yields of protons, neutrons, tritons, ${ }^{3} \mathrm{He},{ }^{4} \mathrm{He}$ and the sum of yields of all nuclei with charges greater than 12 . The temperature range of 3 to $6 \mathrm{MeV}$ are of interest to many experiments. We also show results at $30 \mathrm{MeV}$. The approximation used in the main part of the text is seen to be quite good.

\section{Applications to other areas}

While the main emphasis of this report is on the thermodynamic model for nuclear multifragmentation, the applications of the approach developed in section 2 to other areas will be mentioned in this appendix. In particular, many problems in statistical mechanics can be reformulated in terms of equations 1-5 in that section. Each problem has a different choice for the factor $\omega$ that appears in these equations and a different interpretation of it within the general structure of those equations. We will now illustrate these remarks with some examples.

Let us consider the following parallel between multifragmentation and per- 
mutations, which appear when Fermi-Dirac and Bose-Einstein statistics are included into problems with identical particles. Any permutation can be broken up into cycle classes and this cycle class decomposition is the basis for this parallel. A given permutation of A particles has a specific cycle class decomposition which specifies the number of cycles of length $k$. This number is similar to the number of clusters of size $k$ in a fragmentation. Moreover, the same type of sum rule holds as with clusters. That is, for any given permutation, the total $A$ is equal to the sum of the cycle length times the number of cycles of that length in that specific permutation. The canonical partition function for non-interacting particles such as Fermi-Dirac or Bose-Einstein particles in a box or in a one body potential well such as a harmonic oscillator well has a form given by eq.2 in section $2[85,86,87]$. For identical particles in a box of volume $V$ and a system at temperature $T$, the $\omega$ weight factor for a cycle of length $k$ is that of eq. 6 with the $q_{k}=1$ in that equation for Bose-Einstein particles and $q_{k}=(-1)^{(k+1)}$ for Fermi-Dirac particles. Once the canonical partition function is obtained from the recurrence relation of eq.5, the thermodynamic free energy $F$ can be calculated and all other thermodynamic quantities also follow from $F$. For example, the pressure will have a form involving an expansion in density and quantum volume which gives the quantum corrections to the ideal gas law coming from the symmetrization or anti-symmetrization of the particles. Bose-Einstein particles in a laser trap which is taken as a harmonic oscillator well have also been studied using this approach [87]. Fermions in a well can also be studied as mentioned in [87] and an extended discussion can be found in [88]. Interactions can also be included along with quantum statistics as shown in [18]. Some further observations regarding permutations are as follows. The result of eq. 4 gives the mean number of cycles of length $i$ in terms of the ratio of the two partition functions $A-i$ and $A$, and the $\omega$ factor for that length. Near the Bose-Einstein condensation transition long cycle lengths start to appear and this manifestation of the transition is analogous to the appearance of large clusters around the liquid gas phase transition. The results of eq.4. give the probability of a particular permutation, specified by its $n$ vector, being present. In RHIC collisions many pions are produced and the application of the methods in sec.2 can also be given. For example Bose-Einstein effects associated with thermal pions have been studied in $[89,90]$. For thermal pions at temperature $T$ in a volume $V$ the cycle length $\omega$ factor of eq. 6 is given by,

$$
\left(\frac{V T^{3}}{2 \pi^{2}}\right)\left(\frac{m}{T}\right)^{2}\left(\frac{1}{k^{2}}\right) K_{2}\left[k \frac{m}{T}\right]
$$

Here, $m$ is the mass of the pion and $K_{2}$ is a MacDonald function. The $\omega$ weight factor also appears in expressions concerning the mean number of pions, its fluctuations, and in higher moments of the pion probability distribution. Examples of these connections are: 


$$
\begin{aligned}
\langle N\rangle & =\sum k \omega_{k} \\
\left\langle N^{2}\right\rangle-\langle N\rangle^{2} & =\sum k^{2} \omega_{k} \\
\left\langle(N-\langle N\rangle)^{3}\right\rangle & =\sum k^{3} \omega_{k} .
\end{aligned}
$$

The sums that appear in eq.C.1 are over all $k$ 's. Note that Poisson statistics has only unit cycles, or $k=1$ only in the sums. Then $\left\langle N^{2}\right\rangle-\langle N\rangle^{2}=\langle N\rangle$. The presence of cycles of length 2 and higher cycles produces departures from Poisson statistics. An important observation related to Poisson statistics comes from the fact that coherent states have associated Poisson distributions. Moreover, departures from Poisson statistics are associated with chaotic emission processes. At high temperatures, Maxwell-Boltzmann statistics apply which leads to Poisson statistics in statistical models. The pion probability distribution for having $N$ pions is the ratio of the canonical partition function for a system of size $N$ divide by the grand canonical partition function. This probability was investigated in [89] for the case of $158 \mathrm{GeV} \mathrm{Pb}+\mathrm{Pb}$ collisions where it is shown to have a Gaussian shape with a width that is about $10 \%$ larger than a Poisson distribution with the same mean number of pions. Many other models of pion and, in general, particle multiplicity distributions can be developed in a similar manner by specifying another form for $\omega_{k}$. Once $\omega_{k}$ is given, all quantities of interest follow. The importance of a phenomenological approach to multiparticle distributions, which is based on known distributions from probability theory, is shown in $[91,92,93,94,95]$. Moreover, a wide range of physical processes can be accommodated using such an approach. A specific and frequently used distribution is the negative binomial distribution where $\omega_{k}=x \frac{t^{k}}{k}$. The symbol $x$ is the negative binomial parameter while $t$ is another parameter that is important in fixing the mean number of pions and its variance: $\langle N\rangle=x \frac{t}{1-t}$ and $\left\langle N^{2}\right\rangle-\langle N\rangle^{2}=\langle N\rangle(1+(\langle N\rangle / x))$. The generalized approach in $[89,90]$ also includes several well known specific probability distributions as special cases of a more general distribution. Here, we will just mention a few examples of various phenomena that can be found in $[89,90]$ which are as follows: (1) Emission from systems with a variable signal to noise ratio, where the signal is related to a Poisson processes which may originate from a coherent state and a noise level given by a negative binomial distribution. (2) Field emission from Lorentzian line shapes and its connection to a Feynman-Wilson gas [96]. (3) Pion laser models [97,98] and the role of Bose-Einstein enhancement for a Poisson emitting source. (4) Multiparticle emission as a one dimensional random walk process along a jet axis. A reader interested in the application of the methods of sect.2 to multiparticle multiplicity distributions can find the details and several other individual cases in $[89,90]$. In a series of papers $[85,86]$, Hegyi has considered many interesting aspects of multiparticle production and has also introduced a generalized distribution for its description. 
Photon count distributions can also be developed using the approach of section 2. In fact, early models of pionic distributions [91] coming from nucleonnucleon and nucleus-nucleus collisions were based on photon count distributions [91]. The laser distribution of [91] is an example of a distribution which first appeared in quantum optics and was then subsequently taken over into the area of particle production. Thermal emission of photons have an $\omega_{k}$ factor that can be obtained as the zero mass limit of the pion result given above; namely $\omega_{k}=2 V T^{3} /\left(\pi^{2} k^{4}\right)$. An additional factor of 2 appears for the spin of the photon.

\section{References}

[1] J. Hubele et al., Z. Phys. A 340 (1991) 263.

[2] F. Reif, Fundamentals of statistical and thermal physics, (McGraw-Hill, New York,1965)chap. 8.

[3] A. Z. Mekjian, Phys. Rev. Lett. 38 (1977) 640.

[4] J. Gosset, J. I. Kapusta and G. D. Westfall, Phys. Rev. C 18 (1978) 844.

[5] S. Das Gupta and A. Z. Mekjian, Phys. Rep 72 (1981) 131.

[6] J. Randrup and S. E. Koonin, Nucl. Phys. A 471 (1987) 355c.

[7] D. H. Gross and H. Massmann Nucl. Phys. A 471 (1987) 339c.

[8] J. P. Bondorf, A. S. Botvina, A. S. Iljinov, I. N. Mishustin and K. Sneppen, Phys. Rep. 257 (1995) 133.

[9] S. Das Gupta and A. Z. Mekjian, Phys. Rev. C 57 (1998) 1361.

[10] H. R. Jaqamann, A. Z. Mekjian and L. Zamick, Phys. Rev. C 27 (1983) 2782.

[11] M. W. Curtin, H. Toki and D. K. Scott, Phys. Lett B 123 (1983) 289.

[12] G. F. Bertsch and P. J. Siemens, Physics Lett. B 126 (1983) 9.

[13] S. Das Gupta, A. Z. Mekjian and M. B. Tsang, Advances in Nuclear Physics 26 (2001) 89 (Kluwer Academic/Plenum Publishers, New York).

[14] P. Bhattacharyya, S. Das Gupta and A. Z. Mekjian, Phys. Rev. C 60 (1999) 054616 .

[15] K. C. Chase and A. Z. Mekjian, Phys. Rev. C 52 (1995) R2339.

[16] S. R. Souza et al., Phys. Rev. C 67 (2003) 051602.

[17] L. G. Moretto et al., Phys. Rep. 287 (1997) 249.

[18] B. K. Jennings and S.Das Gupta, Phys. Rev. C 62(2000)014901 
[19] W. Bauer, Phys. Rev. C 38 (1988) 1297.

[20] X. Campi, Phys. Lett B 208 (1988) 351.

[21] J. Pan and S. Das Gupta, Phys. Rev. C 51 (1995) 1384.

[22] S. Das Gupta, J. Pan, J. Kvasnikova, and C. Gale, Nucl. Phys. A 621 (1997) 897.

[23] K. A. Bugaev, M. I. Gorenstein, I. N. Mishustin and W. Greiner, Phys. Rev. C $62(2000) 044320$.

[24] J. P. Bondorf, R. Donangelo, I. M. Mishustin and H. Schulz, Nucl. Phys. A 444 (1985) 460

[25] D. Stauffer and A. Aharony, Introduction to Percolation Theory(Taylor and Francis, Washington DC, 1992) ch.2.

[26] B. Widom, J. Chem. Phys. 43 (1965) 3898.

[27] M. E. Fisher, Physics 3 (1965) 255.

[28] J. B. Elliott et al.,Phys. Lett. B 381 (1996) 35.

[29] J. B. Elliott et al., Phys. Lett. B 418 (1998) 34.

[30] R. P. Scharenberg et al., Phys. Rev. C 64 (2001) 054602.

[31] F. Gulminelli and Ph. Chomaz, Phys. Rev. Lett. 82 (1999) 1402.

[32] C. B. Das, S. Das Gupta and A. Majumder, Phys. Rev C 65 (2002) 034608.

[33] M. L. Gilkes et al., Phys. Rev. Lett. 73 (1994) 1590.

[34] T. Lefort et al., Phys. Rev. C 64 (2001) 064603.

[35] A. Ruangma et al.,Phys. Rev. C 66, (2002) 044603

[36] C. B. Das et al., Phys. Rev. C 66 (2002) 044602.

[37] A. L. Goodman, J. I. Kapusta and A. Z. Mekjian, Phys. Rev. C 30 (1984) 851.

[38] J. B. Elliott et al., Phys. Rev. Lett. 85 (2000) 1194.

[39] J. E. Finn et al., Phys. Rev. Lett. 49 (1982) 1321.

[40] J. Hufner and D. Mukhopadhyay, Phys. Lett. B173 (1986) 373.

[41] L. Oddershede, P. Dimon and J. Bohr, Phys. Rev. Lett 71 (1993) 3107.

[42] J. Pan, S. Das Gupta and M. Grant, Phys. Rev. Lett. 80 (1998) 1182.

[43] Ph. Chomaz and F. Gulminelli, Phys. Lett. B 447 (1999) 221.

[44] S. Das Gupta and S. K. Samaddar in "Isospin Physics in Heavy-Ion Collisions at Intermediate Energies" edited by Bao-An Li and W. Udo Schroder (Nova Science Publishers, Inc, Huntington, New York, 2001) ch.4. 
[45] H. Muller and B. D. Serot, Phys. Rev. C 52 (1995) 2072.

[46] H. Muller and B. D. Serot in "Isospin Physics in Heavy-Ion Collisions at Intermediate Energies" edited by Bao-An Li and W. Udo Schroder (Nova Science Publishers, Inc, Huntington, New York, 2001) ch.3.

[47] P. Bhattacharyya, S. Das Gupta and A. Z. Mekjian, Phys. Rev. C 60 (1999) 064625.

[48] C. B. Das, S.Das Gupta and A. Z. Mekjian, Phys. Rev. C 67 (2003) 064607.

[49] C. B. Das, S. Das Gupta and A. Z. Mekjian, Phys. Rev. C 68 (2003) 014607.

[50] C. B. Das, S. Das Gupta and A. Z. Mekjian, Phys. Rev. C 68 (2003) 031601(R).

[51] S. J. Lee and A. Z. Mekjian, Phys. Rev. C 63 (2001) 044605

[52] S. J. Lee and A. Z. Mekjian, Phys. Rev. C 68 (2003) 014608

[53] S. J. Lee and A. Z. Mekjian, Phys. Lett. B 580(2004)137

[54] D. H. Gross, Phys. Rep. 279 (1997) 119.

[55] P. Chomaz, V. Duflot and F. Gulminelli, Phys. Rev. Lett. 85 (2000) 3587.

[56] L. G. Moretto, J. B. Elliott, L. Phair, and G. Wozniak, Phys. Rev. C 66 (2002) 041601(R).

[57] W. P. Tan et al., Phys. Rev. C 68, (2003) 034609.

[58] W.P. Tan, PhD Thesis, Michigan State University (2002).

[59] Z. Chen and C. K. Gelbke, Phys. Rev. C 38, (1998) 2630-2639.

[60] R.J. Charity et al., Nucl. Phys. A483, (1998) 371; R.J. Charity, M. Korolija, D.G. Sarantites, and L.G. Sobotka, Phys. Rev. C 56, (1997) 873; R.J. Charity, ibid. 58, (1998) 1073.

[61] W. Hauser and H. Feshbach, Phys. Rev. 87, (1952) 366.

[62] T. X. Liu et al., Phys. Rev. C 69, (2004) 014603.

[63] M. B. Tsang, W. A. Friedman, C. K. Gelbke, W. G. Lynch, G. Verde, and H. S. Xu Phys. Rev. Lett. 86, 5023 (2001).

[64] M. B. Tsang et al., Phys. Rev. C 64, (2001) 054615.

[65] H. Johnston et al., Phys. Rev. C 56, (1997) 1972.

[66] D. V. Shetty et al., Phys. Rev. C 70, (2004) 011601.

[67] Y. G. Ma et al., Phys. Rev. C 69, (2004) 064610.

[68] S. R. Souza, R. Donangelo, W. G. Lynch, W. P. Tan, and M. B. Tsang, Phys. Rev. C 69, (2004) 031607.

[69] M. Veselsky, G. A. Souliotis, and S. J. Yennello, Phys. Rev. C 69, (2004) 031602. 
[70] A. Ono, P. Danielewicz, W. A. Friedman, W. G. Lynch, and M. B. Tsang, Phys. Rev. C 68, (2003) 051601.

[71] A. S. Botvina, O. V. Lozhkin, and W. Trautmann, Phys. Rev. C 65, (2002) 044610 .

[72] M. B. Tsang et al., Phys. Rev. Lett. 92, (2004) 062701.

[73] H. S. Xu et al., Phys. Rev. Lett. 85, (2000) 716.

[74] M. Veselsky, G. A. Souliotis, and M. Jandel, Phys. Rev. C 69, (2004) 044607.

[75] W. A. Friedman, Phys. Rev. C 69, (2004) 031601.

[76] C. B. Das, S. Das Gupta, X. D. Liu, and M. B. Tsang Phys. Rev. C 64, (2001) 044608 .

[77] S. Albergo et al., Nuovo Cimento A89, (1985) 1.

[78] M. B. Tsang, W. G. Lynch, H. Xi, and W. A. Friedman Phys. Rev. Lett. 78, (1997) 3836.

[79] H. Xi, W. G. Lynch, M. B. Tsang, W. A. Friedman, and D. Durand Phys. Rev. C 59, (1999) 1567.

[80] H. Xi et al. Phys. Rev. C 57, (1998) R462.

[81] A. Z. Mekjian, Phys. Rev. C 17 (1978) 1051.

[82] A. Z. Mekjian, Nucl. Phys. A 384 (1982) 492.

[83] P. Koch, B. Muller and J. Rafelski, Phys. Rep. 142 (1986) 167.

[84] M. Sobel, P. J. Siemens, J. P. Bondorf and H. A. Bethe, Nucl. Phys. A 251 (1975) 502.

[85] K .C.Chase and A.Z.Mekjian, Phys. Rev. C 49 (1994) 2164.

[86] A.Z.Mekjian and S.J.Lee, Phy. Rev. A 44 (1991) 6294.

[87] K.C.Chase, A.Z.Mekjian and L.Zamick, European Phys. J. B 8 (1999) 281.

[88] S.Pratt, PRL 84 (2000) 4255.

[89] A.Z.Mekjian, B.Schlei and D.Stottman, Phys. Rev. C 58 (1998) 3627.

[90] S.J.Lee and A.Z.Mekjian, Nucl. Phys. A 730 (2004) 514.

[91] P.Carruthers and C.C.Shih, Int. J. Mod. Phys. A2 (1987) 1447.

[92] I.M.Dremin and J.W.Gary, Phys. Rep. 349 (2001) 301.

[93] E.A.Wolf, I.M.Dremin and W.Kittel, Phys. Rep. 270 (1996) 1.

[94] P.Bozek,M.Ploszajczak and R.Botet, Phys. Rep. 252 (1995) 101.

[95] S.Hegyi, Phys. Lett. B 309 (1993) 443, B 318 (1993) 642, B 327 (1994) 171. 
[96] A.Z.Mekjian, Phys. Rev. C 65 (2002) 014907.

[97] S.Pratt, Phys. Lett. B 301 (1993) 159.

[98] T.Csorgo and J.Zimanyi PRL 80 (1998) 916.

[99] J.Klauder and E.Sudarshan, Fundamentals of Quantum Optics, Benjamin, N.Y. 1968. 